$1-1-1903$

\title{
Commercial fertilizers : report for 1903.
}

\author{
J. H. Stewart
}

B. H. Hite

Follow this and additional works at: https://researchrepository.wvu.edu/ wv_agricultural_and_forestry_experiment_station_bulletins

\section{Digital Commons Citation}

Stewart, J. H. and Hite, B. H., "Commercial fertilizers : report for 1903." (1903). West Virginia Agricultural and Forestry Experiment Station Bulletins. 91.

https://researchrepository.wvu.edu/wv_agricultural_and_forestry_experiment_station_bulletins/91 
Digitized by the Internet Archive in 2010 with funding from

Lyrasis Members and Sloan Foundation 


\title{
WEST VIRGINIA UNIVERSITY AGRICULTURAL EXPERIMENT STATION, MORGANTOWN, W. VA.
}

\section{Commercial Fertilizers.}

\section{REPORT FOR 1903.}

\author{
PART I. \\ From January 1st, to May 1st.
}

By J. H. STEWART and B. H. HITE.

[The Bulletins and Reports of this Station will be mailed free to any citizen of West Virginia upon written application. Address, Director of Agricultural Experiment Station, Morgantown, W. Va.] 


\section{THE REGENTS OF THE WEST VIRGINIA UNIVERSITY}

NAME OF REGENT.

P. O. ADDRESS.

Hon. W. J. W. Cowden, .................. Wheeling How. C. MI. BABB, ....................... Falls

Hon. J. B. Finley, .................... Parkersburg Hon. D. C. Gallaher, ................... Charleston Hon. E. M. GRANT, ..................... Morgantown Hon. J. M. HaLE, . . . . . . . . . . . . . Princeton Hon. C. §. Haworth, ................... Huntington Hon. C. R. Oldham, . . . . . . . . . . . . . . . . Moundsvilie

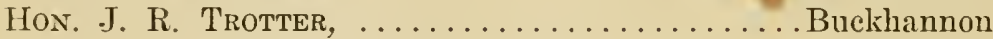

President of the Board of Regents, .........W. J. W. Cowden President of the University,.............. B. Purinto.

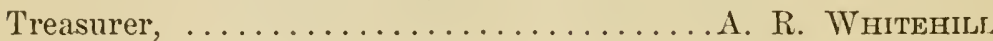

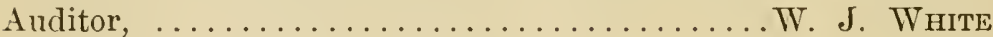

\section{STATION STAFF.}

James H. Stewart, A. MI.,........ Director and Agriculturist Bert H. Hite, M. S.,........... Vice Director and Chemist John L. Sheldon, Ph.D., ............... Plant Pathologist W. E. Rumisey, B. S. Agr.,........... Entomologist in Charge HorACE Atwood, M. S. Agr.,......... AssistantAgriculturist Chas. D. Howard, B. S.,...............Associate Chemist 'T'. C. Johnson, MI. S.,... . Botanist and Assistant Horticulturist

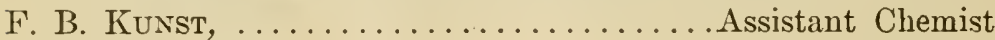
Gilbert M. JoHn, ..............Assistant Horticulturist

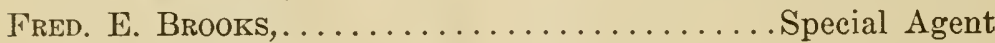
W. J. Wніте, . . . . . . . . . . . . . . Bookkeeper

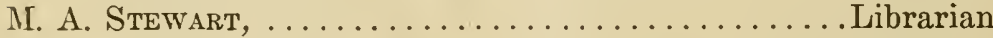
MaY Watson, $\ldots \ldots \ldots \ldots \ldots \ldots \ldots \ldots$ Stenographer 


\section{REPORT ON FERTILIZER INSPECTION.}

The following pages are a report of the work of this office in the execution of the statute of the State of West Virginia, concerning Commercial Fertilizers for the first half of the calendar year, 1903. A supplementary report eovering the work of the latter half of the year will be issued soon.

\section{GOODS OF}

\section{AMFRICAN AGRICULTURAL CHEMICAL COMPANT,}

New York AND Baltimore.

4782. REGULAR CORN FERTILIZER. J. L. Woodyard, Pruntytown. Guarantee: Phosphoric acid soluble, $t$; reverted, 2 ; insoluble, 1 ; total 7 ; available, 6 ; potash, 3 ; phosphoric acid from dissolved phosphate rock; potash from kainit. Found: Phosphoric acid, soluble, 1.19; reverted, 5.72; insolıbie, 0.93 ; total, 7.84 ; available, 6.91 ; potash, 3.40 ; *chlorme, 4.7.

*This represents the proportion of chlorine corresponding to one li $\in \mathrm{m}$ cent of potash in the fertilizer. The ractual percentage of chlo. rine may be found by multiplying the above by the actual percentage "f potash present. Chlorine is a constituent of muriate of potash, of common salt and of certain compounds of lime and magnesia. Then high glade sulphate is used as the source of potash the fertilizer should contain no more than traces of chlorine. The quantity of the latter increases directly with the inferiority of the source employed and its estimation serves approximately as an indication of such source. Where lainit is guaranteed and the chlorine exceeds 4.5, it may be assumed that an inferior grade of this material has been used. (See, page 52.) 
4792. REGULAR CORN FERTILIZER. J. M. Miller \& Bro., Roneeverte. Guarantec-(As above.) Found: Phosphoric aciri, soluble, 0.86; reverterl, 6.00; insoluble, 0.82 ; total, 7.65; :vailahle, 6.86 ; potash, 2.56 ; chlorine, 5.2 .

4698. REGULAR CORN FERTILIZER. Washington and Alexander, Charles Town. Guarantee,-(As above.) Found: Phosphoric aeid, soluble, 1.39 ; reverted, 5.78; insoluble, 0.77; total, 7.94 ; available, 7.17 ; potash, 3.40 ; ehlorine, 4.4 .

4718. SUPERPHOSPIIATES. Griffith \& Howell, Belington. Guarantee: Phosphoric acid, soluble, 14.00 ; reverted, $\stackrel{-}{-}$ insoluble, 1; total, 17 ; available, 16.00; phosphoric acid from dissolved phosphate rock. Found: Phosphoric acid, soluble, 14.30 ; reverted, 3.47 ; insoluble, 0.29 ; total, 18.06 ; availabir, 17.77

4694. GEM ALTALINE BONE. R. Hunter, Berkeicy Springs. Guarantee: Phosphorie acid, soluble, 4.00 ; reverted, 2.00 ; insoluble, 1.00 ; total, 7.00 ; available, 6.00 ; potash, 3.00 ; phosphoric acid from dissolved phosphate rock; potash from kainit. Found: Phosphoric acid, soluble, 7.60 ; reverted, 5.27; insoluble, 0.79 ; total, 7.66 ; available, 6.87 ; potash, 3.23 ; chlorine 4.8 .

4759. GEM ALKALINE BONE. T. B. Drummond \& Co., Buckhannon. Guarantee-(As above.) Found: Plosphorie acid, soluble, 1.14; reverted, 5.70; insoluble, 0.70 ; total, 7.54; available, 6.84 ; potash, 3.13 ; chlorine, 3.5 .

4730. GEII ALKALINE BONE. Deberry \& Bishop, Terra Alta. Guarantee-(As above.) Found: Phosphoric acil, soluble, 1.43 ; reverted, 5.88 ; insoluble, 0.86 ; total, 8.17 ; available, 7.31 ; potash, 2.86 ; chlorine, 3.3 .

4722. GEM ALKALINE BONE. Griffith a Howell, Bilington, Guarantec-(As above.) Found: Phosphoric ack\}, 
soluble, 1.98 ; revertéd, 4.80 ; insoluble, 0.72 ; total, 7.50 ; avaiiable, 6.78 ; potash, 3.01 ; chlorine, 3.3.

4742. PURE GROUND BONE. J. H. Miller \& Sons, Martinsburg. Guarantee: Total phosphoric acid, 20.00; nitrogen, 3.29; from animal bones. Found: Total phosphoric acid, 20.67; nitrogen, 3.89 ; *availability of nitrogen, 78 .

4758. BONE MEAL. T. B. Drummond \& Co., Bucl:hannon. Guarantee: Total phosphoric acid, 14.00 ; nitrogen, 1.65; from animal bones. Found: Phosphoric acid, totil, 17.04; nitrogen, 1.56; availability of nitrogen, 87.

4661. CANTON CHEMICAL BAKER'S DISSOLVED S. C. BONE. J. M. Hagerty, Farmington. Guarantee: Phosphoric acid, soluble, 12.00 ; reverted, 2.00 ; insoluble, 1.00 ; total, 1500 ; available, 14.00; from dissolved phosphate rock. Found: Phosphoric acid, soluble, 11.16 ; reverted, 4.34; insoluble, 1.37; total, 16.77 ; available, 15.40.

4700. CANTON CHEHICAL BAKER'S DISSOLVED S. C. BONE. Washington \& Alexander, Charles Town. Guaruntee-(As above.) Found: Phosphoric acid, soluble, 10.69; reverted, 4.74 ; insoluble, 1.15 ; total, 16.58 ; available, 15.42.

4774. CANTON CHEMICAL BAKER'S DISSOLVED S. C. BONE. C. W. Siever, Keyser. Guarantee-(As above.) Found: Phosphoric acid, soluble, 10.93; reverted, 2.98 ; insoluble, 1.25 ; total, 15.16 ; available, 13.91.

*The figure for the availability of nitrogen represents that proportion of the total nitrogen of the fertilizer which $i_{S}$ immediately avai:able to the plant as food The test employed is a modification of Sitreet's neutral permaganate method. While the results obtained can be considerend as but an approximation to the true quantities as inclicated by actual field tests, nevertheless they are valuable as serving to show the relative availability of the nitrogen in various wiands of fertilizers. An availability of 85 or above $i_{s}$ considerert high 80-85 medium, below 75 , $10 \mathrm{w}$. The availability of nitrogen in bone meats is necessarily higher than in mixed goods unless nitrate is used in the latter. 
4736. CANTON CHEMICAL BAKER'S DISSOLVED \&. ('. BONE. L. P. Licklider, Martinsburg. Guarantec-(As above.) Found: Phosphoric aeid, soluble, 13.32; reverted, 2..21; insoluble, 0.82; total, 16.35; available, 15.53 .

4763. CANTON CHEMHCAL BAKER'S DISSOLVEI) $\therefore$ ('. BONE. Arunstrong, Crislip \& Co., Buckhannon. Gua)antee-(As above.) Found: Phosphoric acid, soluble, 6.08; reverted, 10.07 ; insoluble, 0.90 ; total, 17.05 ; available, 16.15 .

4663. CANTON CHEMICAL SOLUBLE BONE AND FOT_1SI. J. M. Hagerty, Farmington. Guarantee: Phosphoric, acil, soluble, 8.00 ; reverted, 2.00 ; insoluble, 1.00 total, 11.00 ; available, 10.00 ; potash, 2.00 ; phosphoric acid from dissolved phosphate rock; potash from kainit. Found: Phosphoric acid, soluble. 7.71 ; reverted, 3.18 ; insoluble. 0.74 ; total, 11.68 ; available, 10.89 ; potash, 2.17 ; chlorine, 5.3.

4766. CANTON CHEMHCAL SOLUBLE BONE ANI I'OTASII. Armstrong, Chrislip \& Co., Buckhannon. Guarantee-(As above.) Found: Phosphoric acid, soluble, 4.70; reverted, 5.81 ; insoluble, 0.72 ; total, 11.23 ; available, 10.51 ; potash, 2.12 ; chlorine, 5.1 .

4786. CANTON CIIEMICAL SOLUBLE BONE ANT) POTASH. C. W. Siever, Keyser, Guarantce-(As above.) Found: Phosphoric acid, soluble, 5.95; reverted, 4.17; insoub)e, 1.46; total, 11.58; available, 10.12; potash, 1.71; chloline, 4.7 .

4662. CANTON CIIEMICAL HARROW BRAND CROI' (ikOUER. J. M. Hagerty, Farmington. Guarantec: Phosphoric acid, soluble, 6.00; reverted, 2.00; insoluble. 1.00; total, 9.00 ; a vailable, 8.00 ; nitrogen, 0.82 ; potash, 1.00 ; phosphoric acid from dissolved phosphate rock and animal bone; nitrogen from dissolved fish, tankage and other animal matter; potash 
from kainit. Found: Phosphoric acid, soluble, 7.07 ; reverted, 1.98; insoluble, 1.0t; total, 10.09 ; available, 9.05 ; nitrogen, 0.95; potash, 1.24; chlorine, 8.1; availability of nitrogen, 80 .

\section{CANTON CHEMICAL HARROW BRAND CROP} GROWER. L. P. Licklider, Martinsburg. Guarantee-(As above.) Found: Phosphorie acid, soluble, 4.36; reverted 3.37; insoluble, 0.64 : total, 8.37 : available, 7.73 ; nitrogen, 0.93 ; potas'?, 1.17 ; chlorine, 6.6 ; availibility of nitrogen, 80 .

4664. CANTON CHEMICAL POTATO MANURE. J. II. Hagerty, Farmington. Guarantee: Phosphoric acid, soluble, 4.00; reverted, 2.00. insoluble, 1.00 ; total, 7.00 ; available, 6.00 ; nitrogen, 1.23; potash, 5.00; phosphoric acd from dissolved phosphate rock; nitrogen from dissolved fish, tankage and other animal matter; potash from kainit and manure salts. Found: Phosphoric acid, soluble, 3.95 ; reverted, 2.88; insoluble, 1.19; total, 8.02; available, 6.83; nitrogen, 1.27; potash, 5.04; chlorine, 3.2 ; availibility of nitrogen, 80 .

4701. CANTON CHEMICAL POTATO MANURE. Washington \& Alexander, Charles Town. Guarantee-(As above.) Found: Phosphoric acid, soluble, 4. 41; reverted, 2.29; insoluble, 1.27; total, 7.97; available, 6.70; nitrogen, 1.22; potash, 4.67 ; chlorine, 2.8 ; availability of nitrogen, 83.

4767. CANTON CHEMICAL POTATO MANURE. Armstrong, Chrislip \& Co., Buckhannon. Guarantee-(As above.) Found: Phosphoric acid, soluble, 4.22; reverted, 2.80; insoluble, 0.99 ; total, 8.01 ; available, 7.02 ; nitrogen, 1.33 ; potash, 5.18; chlorine, 1.9; availability of nitrogen, 84 .

4785. CANTON CHEMICAL POTATO MANURE. C. W. Seiver, Keyser. Guarantee-(As above.) Found: Phosphoric acid, soluble, 2.48; reverted, 3.55; insoluble, 0.69): total, 6.72 ; available, 6.03 ; nitrogen, 1.40 ; potash, 4.86 ; chlí rine, 3.1 ; availability of nitrogen, 82 . 
4665. CANTON CHEMICAL GAME GUANO. J. MI. Hagerty, Farmington. Guarantee: Phosphoric acid, solubie, 6.00 ; reverted, 2.00 ; insoluble, 1.00 total, 9.00 available, 8.00 ; nitrogen, 1.65; potash, 2.00; phosphoric acid from dissolved phosphate rock and animal bones; nitrogen from dissolved fish, tankage and other animal matter; potash from kainit. Found: Phosphoric acid, soluble, 6.37; reverted, 2.11; insoluble, 1.4:; total, 9.90 ; available, 8.58 ; nitrogen, 2.08; potash, 2.42; chlorine, 3.3 ; availability of nitrogen, 88 .

4666. CANTON CHEMICAL BAKER'S SPECIAL WHEAT, CORN AND GRASS MITTURE. J. M. Hagert: Farmington. Guarantee: Phosphoric acid, soluble, 7.00 ; reverted, 2.00; insoluble, 1.00 ; total, 10.00 ; available, 9.00 ; nitrogen, 0.82 ; potash, 2.00; phosphoric acid from dissolved pho: phate rock and animal bones; nitrogen from dissolved fish, tanls. age and other animal matter; potash from kainit. Found: Phosphoric acid, soluble, 7.10 ; reverted, 2.63; insoluble, 1.30; total, 11.03 ; available, 9.73 ; nitrogen, 1.16 ; potash, 1.94 ; chlorine, 3.8 ; availability of nitrogen, 83.

4696. CANTON CHEMICAL BAKER'S SPECIAL WHEAT, CORN AND GRASS GROWER. W. H. Brady, Berkelcy Springs. Guarantee-(As above.) Found: Phosphoric acid, soluble, 7.29 ; reverted, 1.73 ; insoluble, 1.65 ; total, 10.67 ; available, 9.02 ; nitrogen, 0.91 ; potash, 2.04 ; chlorine, 3.9 ; availability of nitrogen, 80 .

4793. CANTON CIEEMICAL BAKER'S SPECLAL THEAT, CORN AND GRASS MITTURE. Miller, Roncevertc. Guarantce-(As above.) Found: Phosphoric aciel, soluble, 6.92 ; reverted, 1.77 ; insoluble, 1.89 ; total, 10.58 ; availahle, 5.69 ; nitrogen, 1.08 ; putash, 2.00 ; chlorine, 3.3 ; availaibility of nitrogen, 85 .

4699. CANTON CHEIICAL BAKER'S SOLUBLE AL- 
KALINE BONE. Washington \& Alexander, Charles Town. Guarantee: Phosphoric acid, soluble, 10.00; reverted, 2.00 ; insoluble, 1.00 ; total, 13.00 ; available, 12.00 ; potash, 3.00 ; phòsphoric acid from dissolved phosphate rock; potash from kainit. Found: Phosphoric acid, soluble, 8.51; reverted 3.90; insoluble, 1.38; total, 13.79; available, 12.41; potash, 2.76 ; chlorine, 1.7.

\section{CANTON CHEIICAL EAGLE PHOSPHATE.} L. P. Licklider, Nartinsburg. Guarantee: Phosphoric acid, soluble, 5.00 ; reverted, 2.00 ; insoluble, 1.00 ; total, 8.00 ; available, 7.00 ; nitrogen, 0.82 ; potash, 1.00 ; phosphoric acid from dissolved phosphate rock and animal bone; nitrogen from dissolved fish, tankage and other animal matter; potash from kainit. Found: Phosphoric acid, soluble, 4.02; reverted, 3.92 ; insoluble, 0.91 ; total, 8.85 ; available, 7.94 ; nitrogen, 1.01 ; potash, 1.21 ; chlorine, 7.1 ; availability of nitrogen, 78 .

4773. FINE GROUND BONE. C. W. Siever, Keyser. Guarantee: Total phosphoric acid, 23.00; nitrogen, 2.47; from animal bone. Found: Total phosphorie acid, 25.65; nitrogen, 2.66; availability of nitrogen, 93 .

4702. KAINIT'. Washington \& Alexander, Charles Town. Guarantee: Potash, 12.00. Found: Potash, 11.12.

4712. BRADLEY'S BEAN AND POTATO. J. W. Kinger \& Sons, Terra Alta. Guarantee: Phosphoric acid, solıble, 6.00 ; reverted, 2.00 ; insoluble, 1.00 ; total, 9.00 ; available, 8.00; nitrogen, 0.82 ; potash, 4.00 ; phosphoric acid from dissolved phosphate rock and animal bone; nitrogen from dissolvet fish, tankage and other animal matter; potash from manure salts and kainit. Found: Phosphoric acid, soluble, 4.72; revetred, 3.97 ; insoluble, 0.47 ; total, 9.60 ; available, 8.69 ; nitrogen, 0.87 ; potash, 3.86 ; ehlorine, 3.1 ; availability of nitrogen, 77.

4713. BRADLEY'S ALKALINE BONE. J. W. Ringer 
\& Son, Terra Alta. Guarantec: Phosphoic acid, soluble, o; reverted, 2; insoluble, 1 ; total 11 ; available, 10 ; potash, 2 ; phosphoric acid from dissolved phosphate rock; potash from kainit. I'ound: Phosphoric acid, soluble, 6.73 ; reverted, 3.94 ; insoluble, 0.96 ; total, 11.63; available, 10.67 ; potash, 1.86 ; chlorine, 2.1.

4714. BRADLEY'S SOLUBLE DISSOLVED BONE. Thomas Rogers, Rowleshurg. Guarantec: Phosphoric acid, sollible, 12.00 ; reverted, 2.00 ; insoluble, 1.00 ; total, 15.00 ; avallable, 14.00; phosphoric acid from dissolved phosphate rock. Fund: Phosphoric acid, soluble, 12.10; reverted, 3.56; insoluble, 1.00 ; total, 16.96 ; available, 15.66 .

4715. BRADLEY'S NIAGARA PHOSPHATE. Thos. Rogers, Rowlesburg. Guarantee: Phosphoric acid, soluble, 5.00 ; reverted, 2.00 ; insoluble, 1.00 ; total, 8.00 ; available, 7.00 ; nitrogen, 0.82 ; potash, 1.00; phosphoric acid from dissolved phosphate rock and animal bone; nitrogen from dissolved fish, tankage and other animal matter; potash from kainit. Found: Phosphoric acid, soluble, 5.28; reverted, 2.35; insoluble, 1.33; total, 8.96 ; available, 7.63 ; nitrogen, 0.98 ; potash, 1.40 ; chlorine, 6. 6 ; availability of nitrogen, 79 .

4765. CLETELAND DRYER FOREST CITY BUCKEYE. Bishop \& Barbe, Jane Lew. Guarantee: Phosphoric acid, soluble, 7.00 ; reverted, 2.00; insoluble, 1.00 ; total, 10.00); available, 9.00 ; nitrogen, 2.47 ; potash, 2.00 ; phosporic acid from dissolved phosphate rock and animal bone; nitrogen from dissolved fish, tankage and other animal matter; potash from kainit:. Found: Phosphoric acid, soluble, 7.05 ; reverted, 1.51.; iusoluble, 1.34 ; total. 10.90 ; available, 8.58 ; nitrogen, 1.92 ; potash, 2.45; chlorine, 2.1 ; availability of nitrogen, 88 .

4680 DETRICK'S QUICKSTEP BONE PHOSPHATE IOR POTATOES AND TOBACCO. Jas. R. Morris, Gath. Guarantec: Phosphoric acid, soluble, 6.00 ; reverted, 2.00 ; insol- 
lible, 1.00 ; total, 9.00 ; available, 8.00 ; nitrogen, 2.47; potash, 4.00 ; phosphoric acid from disolved phosphate rock and animal bone; nitrogen from dissolved fish, tankage and other animal natter; potash from kainit and manure salts. Found: Phosphoric acid, soluble, 6.24; reverted, 2.83; insoluble, 2.09; total, 11.16 ; available, 9.07 ; nitrogen, 2.51 ; potash, 4.13 ; chlorine, 1.5 ; availability of nitrogen, 80 .

4679. DETRICK'S SOLUBLE.BONE AND POTASII. Jas. R. Morris, Gath. Guarantee: Phosphoric acid, soluble, 2; insoluble, 1.00 ; total, 11.00 ; available, 10.00 ; potash, 2.00 ; phosphoric acid from dissolved phosphate rock; potash from kainit. Found: Phosphoric acid, soluble, 7.64; reverted, 3.40 ; insoluble, 1.27; total, 12.31; available, 11.04; potash, 2.00 ; chlorine, 4.7.

\section{DETRICK'S CORN AND OATS FERTILIZER.} Jas. R. Morris, Gath. Guarantee: Phosphoric acid, soluble, 7.00 ; reverted, 2.00 ; insoluble, 1.00 ; total, 10.00 ; available, 9.00 ; ritrogen, 0.82; potash, 3.00; phosphoric acid from dissolved whosphate rock and animal bone; nitrogen from dissolved fish, trnkage and other aninial matter; potash from kainit and manure salts. Found: Phosphoric acid, soluble, 7.28; reverte.1, 2.16; insoluble, 1.23; total, 10.67 ; available, 9.44 ; nitrogen, 1.13; potash, 3.27 ; chlorine, 3.5 ; availability of nitrogen, 82 .

4724. LAZARETTO HIGH GRADE DISSOLVED BONE PHOSPHATE AND POTASII. Griffith \& Howell, Belington. Guarantee: Phosphoric acid, soluble, 10.00 ; reverted, 2.00 ; irtsoluble, 1.00 ; total, 13.00 ; available, 12.00 potash, 5.00; phosphoric acid from dissolved l'ock; potash from kainit and manure salts. Found: Phosphoric acid, soluble, 9.07; reverted, 3.60; insoluble, 0.80 ; total, 13.47 ; available, 12.67 ; potash, 4.70 ; chlo. ine, 5.1 .

4682. LAZARETTO HIGII GRADE BONE AND I'OTASH. A. G. Chrislip, Philippi. Guarantee-(As above.) Found: Phosphoric acid, soluble, 8.02 ; reverted, 4.77 ; insoiu- 
ble, 0.55 ; total, 13.34 ; available, 12.79 ; potash, 4.89 ; chlrrine, 1.7.

4756. LAZARETTO AMMONATED BONE PHOSPHATE. T. B. Drummond \& Co., Buckhannon. Guarantee: I'hosphoric acid, soluble, 6.00 ; reverted, 2.00; insoluble, 1.00; total, 9.00 ; available, 8.00 ; nitrogen, 0.82 ; potash, 2.00 ; phesphoric acid from dissolved phosphate rock and animal bone; nitrogen from dissolved fish, tankage and other animal matter; potash from kainit. Found: Phosphoric acid, soluble, 3.21; reverted, 5.60; insoluble, 1.88 ; total, 10.69 ; available, 8.81 ; nitrogen, 0.93 ; potash, 2.19 ; chlorine, 3.0 ; availability of nitrogen, 80 .

4760. LAZARETTO BONE COMPOUND. T. B. Drummond \& Co., Buckhannon. Guarantee: Phosphoric acid, soluble, 7.00 ; reverted, 2.00 ; insoluble, 1.00 ; total, 10.00 ; available, 9.00 ; nitrogen, 1.03 ; potash, 3.00 ; phosphoric acid from dissolved phosphate rock and animal bone; nitrogen from dissolved fish, tankage and other animal matter; potash from kainit and manure salts. Found: Phosphoric acid, soluble, 6.35; reverted, 4.10 ; insoluble, 1.52 ; total, 11.97; available, 10.45 ; nitrogen, 1.11; potash, 3.42 ; chlorine, 1.8 ; availability of nitrogen, 78 .

4761. LAZARETTO CROP GROWER. T. B. Drummond \& Co., Buckhamon. Guarantee: Phosphoric acid, soluble, 6.00 ; reverted, 2.00 ; insoluble, 1.00 ; total, 9.00 ; available, 8.00 ; nitrogen, 1.65 ; potash, 2.00 ; phosphoric acid from dissolved phosphate rock and animal bone; nitrogen from dissolved fish, tankage and other animal matter; potash from kainit. Found: Phosphoric acid, soluble, 5.67 ; reverted, 2.51; insolubl', 1.93 ; total, 10.11 ; available, 8.18 : nitrogen, 1.83 ; potash, 1.97; chlorine, 2.4 ; availability of nitrogen, 84 .

4762. LAZARETTO SPECIAL POTATO FERTILIZER. 'J. B. Drummond \& Co., Buckhannon. Guarantee: Phosphoric acid, solublr, 6.00 ; reverted, 2.00; insoluble, 1.00 total, 9.00; 
available, 8.00 ; nitrogen, 0.82 ; potash, 4.00 ; phosphoric acir from dissolved phosphate rock and animal bone; nitrogen from dissolved fish, tankage and other animal matter; potash from kainit and manure salts. Found: Phosphoric acid, solub!e, 4.80; reverted, 2.39; insoluble, 2.19; total, 9.38; available, 7.19; nitrogen, 1.16 ; potash, 3.99 ; chlorine, 2.4 ; availability of nitrogen, 83.

4764. LAZARETTO DISSOLVED BONE PHOSPHATE AND POTASH. T. B. Drummond \& Co., Buckhannon. Guarantee: Phosphoric acid, soluble, 8.00; reverted, 2.00; insolublo, 1.00 ; total, 11.00 ; available, 10.00 ; potash, 2.00 ; phosphoric acid from dissolved phosphate rock; potash from kainit. Found: Phosphoric acid, soluble, 5.82; reverted, 4.44; insoluble, 1.61; total, 11.87 ; available, 10.26 : potash, 2.04 ; chlorine, 3.1.

4783. MARYLAN'D AMMONIATEDD BONE. J. J. Woorlyard, Pruntytown. Guarantee: Phosphoric acid, soluble, 6.00 ; reverted, 2.00 ; insoluble, 1.00 ; total, 9.00 ; available, 8.00; nitrogen, 1.65: potash, 3.00; phosphoric acid from dissolved phospirate rock and animal bone; nitrogen from dissolved fish, tankage and other animal matter; potash from kainit and manure salts. Found: Phosphoric acid, soluble, 3.98 ; reverted, 5.32; insoluble, 2.94 ; total, 12.24 ; available, 9.30 ; nitrogen, 1.76 ; potash, 2.97 ; chlorine, 1.5; availability of nitrogen, 8.7.

4784. MARYLAND BONE SUPERPHOSPHATE. J. I. Woodyard, Pruntytorn. Guarantee: Phosphoric acid, soluble, 8.00 ; reverted, 2.00 ; insoluble, 1.00 ; total, 11.00 ; available, 10.00 ; potash, 2.00; phosphoric acid from dissolved phosphate rock; potash from kainit. Found: Phosphoric acid, soluble, 6.79; reverted, 3.33 ; insoluble, 1.19 ; total, 11.31; available, 10.12; potash, 1.93 ; chlorine, 1.5 .

4741. SUSQUEHANNA SUPERIOR ROCK PHOS. PHATE. J. H. Miller \& Sons, Martinsburg. Guarantee: 
Phosphoric acid, soluble, 12.00; reverted, 2.00 ; insoluble, 1.00; total, 15.00 ; available, 14.00 ; from dissolved phosphate rock. Found: Phosphoric acid, soluble, 10.59 ; reverted, 4.40 ; insoluble, 1.05 ; total, 16.04 ; available, 14.99 .

4743. SUSQUEHANNA TIT PHOSPHATE. J. II. Miiler \& Sons, Martinsburg. Guarantee: Phosphoric acid, solubir, 6.00 ; reverted, 2.00 ; insoluble, 1.00 ; total, 9.00 ; available, 8.00 ; nitrogen, 0.82 ; potash, 1.00 ; phosphoric acid from dissolver whosphoric rock and animal bone; nitrogen from dissolved fish, tankage and other animal matter; potash from kainit. Fonnd: Phosphorie acid, soluble, 4.30; reverted, 3.47 ; insoluble, -1.07 ; total, 9.84 ; available, 7.77 ; nitrogen. 1.15 ; potash, 1.34 ; chlorine, 6.1 ; availability of nitrogen, 83.

4744. SUSQUEHANNA POTATO PHOSPHATE. J. II. Miller \& Sons, Martinsburg. Guarantee: Phosphoric acil, soluble, 6.00 ; reverted, 2.00 ; insoluble, 1.00 ; total, 9.00 ; available, 8.00 ; nitrogen, 1.65 ; potash, 5.00 ; phosphoric acid from (iissolved phosphate rock and animal bone; nitrogen from dinsolved fish, tankage and other animal matter; potash from liainit and manure salts. Found: Phosphorie aeid, soluble, 1.75; reverted, 2.99 ; insoluble, 0.79 ; total, 8.53 ; available, 7.74 ; ritrogen, 1.92 ; potash, 5.01 ; chlorine, 1.9 ; availability of nitrogell, 84.

4684. WILLIAMS' \& CLART'S GOOD GROWER POIAiO PHOSPIIATE. A. G. Chrislip, Philippi. Guarantee: Phosphoric acid, soluble, 4.00; reverted, 2.00; insoluble, 1.00; total, 7.00 ; available, 6.00 ; nitrogen, 1.23 ; potash, 5.00 ; phosphoric acid from dissolved phosphate rock and animal bone; nitrogen from dissolved fish, tankage and other animal mattcr; petash from kainit and manmre salts. Found: Phosphoric acid, soluble, 5.30; reverted, 2.23; insoluble. 0.95; total, 8.48; ivailable, 7.53 ; nitrogen, 1.49 ; potash, 4.86 ; chlorine, 0.2 ; avail. ability if nitrogen, 84 . 
4768. WILIIAMS' \& CLARK'S GOOD GROWER POTATO PHOSPHATE. Bishop \& Barbe, Jane Lew. Guarantee--(As above.) Found: Phosphoric acid, soluble, 2.89; reverted, 3.66; insoluble, 1.45 ; total, 8.00 ; available, 6.55 ; nitrogen, 1.31 ; potash, 5.32 ; chlorine, 2.1 ; availability of nitrogen, 79 .

4719. WILLIAIS' \& CLARK'S ACORN ACID PHOSPHATE. Griffith \& Howell, Belington. Guarantee: Phosphoric acid, soluble, 12.00 ; reverted, 2.00 ; insoluble, 1.00 ; total, 15.00; available, 14.00; from dissolved phosphate rock. Found: Phosphoric acid, soluble, 12.20 ; reverted, 4.16 ; insoluble, 1.13; total, 17.49; available, 16.36 .

4723. WILLIAMS' \& CLARK'S PROLIFIC CROP PRODUCER. Griffith \& Howell, Belington. Guarantee: Phosthorie acid, soluble, 5.00 ; reverted, 2.00 ; insoluble, 1.00 ; total, $\$ .00$; available, 7.00 ; nitrogen, 0.82 ; potash, 1.00 ; phosphor:e acid from phosphate rock and animal bone; nitrogen from dissolved fish, tankage and other animal matter; potash from kainit. Found: Phosphoric aeid, soluble, 4.22; reverted, 2.90; iusoluble, 1.13; total, 8.30; available, 7.12; nitrogen, 1.20); potash, 1.62 ; chlorine, 1.1; availability of nitrogen, 78 .

4676. ZELL'S DISSOLVED S. C. PHOSPHATE. W. F. Sill, Pennsboro. Guarantee: Phosphoric acid, soluble, 10.0); reverted 2.00 ; insoluble, 1.00 ; total, 13.00 ; available, 12.00 ; from dissolved phosphate rock. 'Found: Phosphorie acid, soluble, 7.37; reverted, 5.95; insoluble, 1.14; total, 14.46; availabie, 13.32 .

4692 ZELL'S DISSOLVED S. C. FHOSPHATE. li. IIunter, Berkeley Springs. Guarantee-(As above.) Foun l: Phosphoric acid, soluble, 11.86; reverted, 3.73; insoluble, 1.3i; total, 16.93; available, 15.59 .

4691 ZELL'S ELECTRIC PHOSPHATE. Philippi Mill Co., Philippi. Guarantee: Phosphorie acid, soluble, 8.01); 
reverted, 2.00; insoluble, 1.00; total, 11.00, available, 10.00; potash, 2.00; phosphoric acid from dissolved phosphate rock; potash from kainit. Found: Phosphoric acid, soluble, 7.51; 'ererted, 3.00 ; insoluble, 0.6 ; total, 11.18; available, 10.41. potash, 1.9S; chlorine, 5.7 .

4721. ZELL'S ELECTRIC PHOSPHATE. Benton T'eter, Belington. Guarantee-(As above) Found: Phosphoric acid, soluble, 7.16; reverted. 3.24; insoluble, 0.79; totil, 11.19 ; arailable, 10.40 ; potash, 2.10 ; chlorine, 5.7.

4693. ZELL'S DISSOLVED BONE PHOSPHATE. R. IHunter, Berkeley Springs. Guarantee: Phosphoric aeid, soluble, 12.00 ; reverted, 2.00 ; insoluble, 1.00 ; total, 15.00 ; available, 14.00; from dissolved phosphate rock. Found: Phosphoris acid, soluble, 12.06; reverted, 4.08; insoluble, 1.27; total, 17.31: rvailable, 16.04 .

4729. ZELL'S DISSOLVED BONE PHOSPHATE. DeBerry \& Bishop, Terra Alta. Guarantee-(As above.) Found: Phosphoric aeid, soluble, 12.11; reverted, 4.12; insoluble, 1.32; total, 17.55; available, 16.23.

4795. ZELL'S DISSOLVED BONE PHOSPHATE. Julnson \& îrinn, Alderson. Guarantee-(As above.) Founl: Phosphoric acid. soluble, 11.09 ; reverted, 3.3t; insoluble, 1.46; total, 15.89 , available. 14.43 .

4695. ZELL'S LITTLE GIANT. R. Hunter, Berkeley Springs. Guarantee: Phosphoric acid, soluble, 5.00 ; revertist, 2.0 ?) insoluble, 1.00 ; total, 8.00 ; available, 7.00 ; nitrogen, 0.82 ; potash, 1.00; phosphoric acid from dissolved phosphate rock and animal bone; nitrogen from dissolved fish, tankage and nther animal matter; potash from kainit. Found: Phosphoric reid, soluble, 5.25; reverted, 2.06; insoluble, 1.22; total, 8.53; available, 7.31 ; nitrogen, 0.93 ; potash, 1.64 ; chlorine, 6.4 ; availitbility of nitrogen, 80 . 
4731. ZELL'S LITTLE GIANT. De Berry \& Bishop, Trrra Alta. Guarantee-(As above.) Found: Phosphorie acid, soluble, 4.80; reverted, 2.70 ; insoluble, 1.19; total, 8.69; ivailable, 7.50 ; nitrogen, 1.01; potash, 1.81; chlorine, 3.5; availability of nitrogen, 78 .

4720. ZELL'S AMMONIATED BONE SUPERPHOSPHATE. Benton Teeter, Belington. Guarantee: Phosphorie acid, soluble, 6.00 ; reverted, 2.00 ; insoluble, 1.00 ; total, 9.00 ; arailable, 8.00 ; nitrogen, 1.65 ; potash, 2.00 ; phosphoric aci!!, ciissolved phosphate rock and animal bone; nitrogen, dissolveri fish, tankage and other animal matter; potash from kainit. Fonnd: Phosphoric acid, soluble, 5.58; reverted, 3.11; insolubie, 2.09 ; total, 10.78 ; available, 8.69 ; nitrogen, 1.78 ; potash, 1.98 ; ihlorine, 2.7 ; availability of nitrogen, 87 .

\section{REESE HIGH GRADE POTASH MIXTURE.} W. F. Sill, Pennsboro. Guarantees Phosphoric acid, solubl:, 10.00 ; reverted, 2.00; insoluble, 1.00; total, 13.00; available, 12.00 ; potash, 5.00 ; phosphoric acid from dissolved phosphat? rock; potash from kainit and manure salts. Found: Pho:I. horic acid, solnble, 9.21 ; reverted, 3.29 ; insoluble, 0.75 ; totai, 13.25; available, 12.50 ; potash, 4.93 ; chlorine, 1.7.

\section{ARMOUR FERTILIZER WORKS,}

Chicago, ILl.

4688. HIGH GRADE POTATO. W. H. Bailey Hardware Company, Morgantown, W. Va. Guarantee: I'hosphoric acid, soluble, 6.00; reverted, 2.00; insoluble, 2.00 ; total, 10.00 ; available, 8.00 ; nitrogen, 1.65 ; potish, 10.00; phosphoric acid from animal bones and superphosphates; nitrogen from bone, blood, tankage and nitrate of soda; potash from muriate and sulphate of potash. Found: Phosphoric acid, soluble, 5.49; reverted, 3.05; insoluble, 1.33; total, 
9.87 ; animal, 8.54; nitrogen, 1.63; potash, 10.43; ehlorine, 2.9 ; availability of nitrogen, 89 .

4689. STAR PHOSPHATE. T. H. Bailey Hariware Company, Norgantown, West Virginia. Guarcintec: Phosphoric acid, soluble. 12.00; reverted, 2.01); insoluble, 2.00; total, 16.00 ; available. 14.00: phosphoric acid from superphosphate. Found: Phosphoric acid, solubl., 13.07 ; reverted, 2.64; insoluble, 0.82 ; total, 16.53 ; arailable, 15.71 .

4687. WHEAT, CORN AND OATS SPECIAL. W. II. Bailey Hardware Company, Morgantown, IT. Ta. Gunvantre: Phosphoric acid, soluhle. 5.00; reverted, 2.00: insoluble, 2.00; total, 9.00; available, T.vi); nitrogen. $0 . \$ 2$ : potash. 1.00; phosphoric aeid from animal bone and superphosphate; nitrogen from bone, blood, tankag.? and nitrate of soda; potash from muriate of potash, sulphat: of potash and kainit. Found: Phosphoric acid, soluble, 5.97: reverted, 3.47 ; insoluble, 0.86 ; total, 10.32 ; available, 9.46 ; nitr(i\&Een, 0.82 ; potash, 1.28; chlorine, 2.1 ; availability of nitrogen, 75 .

4690. GRAIN GROTER. W. H. Bailey Hardware Co.. Jorgantown. Guarantee: Phosphorie acid, soluble, 6.00: rererted, 2.00; insoluble, 2.00; total, 10.00 ; available, 8.00 ; nitrocen. 1.65; potash, 2.00; phosphoric aeid from animal bone and superphosphate; nitrogen from bone, blood, tankage and nitrats of soda; potash from muriate of potash, sulphate of potash aud kainit. Found: Phosphoric acid, soluble, 6.40; reverted, 2.02; insoluble, 0.37 ; total, S.79; available, 8.42 ; nitrogen, 1.59 ; potash, 2. 30 ; cllorine, 3.4 ; arailability of nitrogen, 90 .

46S6. BOYE MEAL. T. H. Bailey Hardware Co., Morgantorn. Guarantee: Phosphoric acid, reverted, 1t: insoluble, 10.00; total, 24; available, 10.00 ; nitrogen, 2.t7; from aninul 
bones. Found: Phosphoric acid, total, 22.49; nitrogen, 2.84; svailability of nitrogen, 85.

\section{BAUGH \& SONS COMPANY,}

Baltimore. Md.

4677. BAUGH'S CORN FERTILIZER. Harrisville Mill Co., sampled at Pennsboro. Guarantec: reverted, 3.00 ; total, 11.00 ; available, 8.00; nitrogen, 0.82 ; potash, 4.00; phosphoric acid from animal bones and phosphate rock; nitrogen from tankage and animal bones; potash from kainit and muriate. Found: Phosphoric acid, soluble, 7.18; revertec, 1.59; insoluble, 0.70; total, 9. 47; available, 8.77; nitrogen, 1.01; potash, 3.81 ; chlorine, 2.4 ; availability of nitrogen, 65.

4748. BAUGH'S CORN FERTILIZER. R. T. Lowndes, C.Iarksburg. Guarantee-(As Above.) Found: Phosphoric acid, soluble, 5.09; reverted, 3.93; insoluble, 0.83; total, 9.85 ; available, 9.02 ; nitrogen, 1.05 ; potash, 2.38 ; chlorine, 3.2 ; avai!ability of nitrogen, 80 .

4678. BAUGH'S SOLUBLE ALKALINE SUPERPHO':PTATE. Harrisville Nill Co., sampled at Pennsboro. Guarantee: Phosphoric acid, available, 10.00; potash, 2.00 ; phosphoric acid from phosphate rock; potash from kainit and muriate of potash. Found: Phosphoric acid, soluble, 6. 90; reverted, 3.67; insoluble, 1.16; total, 11.73; available, 10.57; potash 2.15 ; chlorine, 5.5 .

4709. BAUGH'S GENERAL CROP GROWER. Offutt \& Lakin, Terra Alta. Guarantee: Phosphoric acid, insoluble, 1.00 ; total, 9.00 ; available, 8.00 ; nitrogen, 0.82 ; potash, 1.00 ; phosphoric acid from animal bone and phosphate rock; nitrogen from tankage and animal bone; potash from kainit. Found: Phosphoric acid, soluble, 5.41; reverted, 3.74; insoluble, 1.01; total, 10.16 ; available, 9.15 ; nitrogen, 0.90 ; potash, 1.79 ; chlorine, 3.7 ; availability of nitrogen, 76 . 
4740. BACGH'S GENERAL CROP GROTER. L. P. Licklider, Nartinsburg. Guarantee-(As above.) Founa: Phosphoric acid, soluble, 4.61; reverted, 4.22; insoluble, 0.96 ; total. 9.19; arailable, 8.83 ; nitrogen, 0.85 ; potash, 1.91 ; chlorine, :3.5; availability of nitrogen, 76 .

475. BAUGH'S GENERAL CROP GROWER. R. I. Lowndes, Clarksburg. Guarantec-(As above.) Found: I'hosphoric acid, soluble, 5.17; reverted, 4.S7; insoluble, 1.10: total, 10.14; available, 9.04 ; nitrogen, 1.09 ; potash, 1.66 : chlorine, 3.9 ; availability of nitrogen, 80 .

4770. BALGH'S GENERAL CROP GROTIER. R. I'. Bollyard, Fetterman. Guarantce-(As above.) Found: Phosphoric acid, soluble, 5.97; reverted, 2.58; insoluble, 0.90; total, 9.45 ; available, 8.55 ; nitrogen, 0.82 ; potash, 1.32 ; clllorime, 5.2; availability of nitrogen, 72.

4710. BAUGH'S HIGII GRADE ACID PHOSPHATE, 1) R DISSOLTED S. C. ROCK. Offutt \& Lakin, Terra Alta. Ginarantee: Phosphoric acid, arailable, 14.00; from dissolved phosphate rock. Found: Phosphoric acid, soluble, 9.15 ; reverted, 6.23; insoluble, 2.75; total, 18.13; available, 15.38 .

4769. BAUGH'S HIGH GRADE ACID PHOSPHATN, (JR DISSOLVED S. C. ROCK. R. B. Bolyard, Fetterman. ficarantee-(As Above) Found: Phosphoric acid, soluble, 11.65; reverted, 1.62 ; insoluble, 1.65 ; total, 17.92 ; available, $16.2 T$.

4711. BAUGH'S DOUBLE EAGLE MHOSPHATE. Gifutt \& Lakin, Terra Alta. Guarantee: Phosphoric acid, in:(1) uble, 3.00 ; total, 11.00; available, 8.00 ; nitrogen, 1.64; potash, 1.00 ; phosphoric acid from animal bone and phosphate rock; nitrogen from tankage and animal bone; potash from kainit. Found: Phosphoric acid, soluble, 4.77 ; reverted, 4.41 ; insoluble, 
1.37 ; total, 10.55 ; available, 9.18 ; nitrogen, 1.67 ; potash, 1.40 ; chlorine, 3.2 ; availability of Nitrogen, 85.

4746. BAUGH'S DOUBLE EAGLE PHOSPHATL. R. T. Lowndes, Clarksburg. Guarantee-(As above.) Found: Phosphoric acid, soluble, 5.03; reverted, 4.08; insoluble, 2.01; total, 11.12 ; arailable, 9.11 ; nitrogen, 1.94 ; potash, 1.48 ; chırine, 4.1 ; availability of nitrogen, 86 .

\section{BAUGH'S DOUBLE EAGLE PHOSPHATE.} i. B. Bollyard, Fetterman. Guarantee-(As above.) Foum: Phosphoric acid, soluble, 5.76; reverted, 2. 95; insoluble, 0.74; total, 9.45 ; available, 8.71 ; nitrogen, 1.96 ; potash. 1.71 ; chlorine, 3.5 ; availability of nitrogen. 81 .

4739. BAUGH'S POTATO FERTILIZER. L. P. Licklider, Martinsburg. Guarantee: Phosphoric acid, insoluble, 3.00 ; total, 11.00 ; available, 8.00 ; nitrogen, 1.64 ; potash, 2.00 ; phosphoric acid from animal bones and phosphate rock; nitrogen from tankage and animal bones; potash from kainit and inuriate of potash. Found: Phosphoric acid, soluble, 4.73 ; reverted, 3.90 ; insoluble, 1.02 ; total, 9.65 ; available, 8.63 ; nitrogen, 2.04; potash, 2.40; chlorine, 3.7 ; availability of nitrogen, 90 .

4788. BAUGH'S POTATO FERTILIZER. R. B. Bol:yard, Fetterman. Guarantee-(As above.) Found: Phojphoric acid, soluble, 5.49; reverted, 3.05 ; insoluble, 1.33 ; total, 9.97; available, 8.54 ; nitrogen, 1.63 ; potash, 2.48 ; chlorine, 2.9 ; availability of nitrogen, 84 .

4747. BAUGH'S SPECIAL POTATO MANURE. R. T.

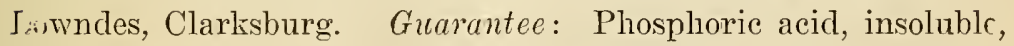
2..00; total, 7.00; available, 5.00; nitrogen. 1.64; potash, 10.00; plısphoric acid from animal bones and phosphate rock: nitrogen from tankage and animal bones; potash from high grade sulphate, kainit, and muriate of potash. Found: Phosphoric 
acir, soluble, 3.56 ; reverted, 4.07 : insoluble 1.11 ; total. 8.74 : available 7.63 ; nitrogen, 1.67 ; potash, 7.39 ; chlorine, 1.0 ; availability of nitrogen, 83 .

4787. BAUGI'S CORN FERTILIZER. R. B. Bollyar!, Fetterman. Guaruntee-(As above.) Found: Phosphorie acid, soluble, 0.91; reverted, 7.57; insoluble, 0.54; total, 9.02: available, 8.48 ; nitrogen, 1.09 ; potash, 4.21 ; chlorine. 2.6 ; availiilility of nitrogen, 83 .

\section{BOWKER FERTILIZER COMPANT,}

Cincinniti, Ohio.

4757. BOWKER'S HARTEST BONE. 'Y' B. Drummond \& Co., Buckhannon. Guarantec: Phosphorie acid, soluble, 2.00 : total, 10.00 ; available, 8.00 ; nitrogen, 0.75 ; potash, 1.00 ; phosphoric aed from dissolved animal bone and mineral phosphate; nitrogen from dissolved bone and tankage: potash from muriate of potash. Found: Phosphoric acid, soluble, 0.81; recerted, 6.60 ; insoluble, 4.14 ; total, 11.55 ; available, 7.41 ; nitroin, 0.84 ; potash, 1.45 ; chlorine, 0.8 ; avalability of nitrogen, 72 .

4775. BOWKER'S IIARVEST BONE. J. E. Lourle?milk, Ronceverte. Guarantec-(As above.) Found: Phosphoric acid, soluble, 5.09; reverted, 3.94: insoluble, 2.75; tota!, 11.78; available, 9.03 ; nitrogen, 0.93 ; potash, 1.19 ; chlorine, 1.6 ; ¿vailability of nitrogen, 84 .

4777. BOWKER'S BONE MEAL. J. E. Tondermili, Roneeverte. Guarantec: Total phosphoric acid, 20.00; nitrogen, 1.50 ; from grotund animal bone; total phosphoric acid, 24.17 ; niтrogen, 1.76 ; availability of nitrogen, 85 .

4780. BOWKER'S SOLLBLE BOVE: J. E. Loudermilk, Ponceverte. Guarantec: Phosphoric acid, insoluble, 2.00 ; totai, 16.00 ; available, 14.00 ; from dissolved mineral phospliat?. 
Found: Phosphoric acid, soluble, 8.51 ; reverted, 5.89 ; insolubl-., 3.83 ; total, 18.23 ; available, 14.44 .

4794. BOTKER'S DISSOLVED BONE AND POTASFi. J. E. Loudermilk, Ronceverte. Guarantee: Phosphoric acid, insoluble, 2.00; total, 12.00; available, 10.00; potash, 2.00; phosphoric acid from dissolved mineral phosphate: potash from muriate of potash. Found: Phosphoric acid, soluble, 5.40; rererted, 3.46 ; insoluble, 2.70; total, 11.56; available, 8.86; potasin, 1.86 ; chlorine, 3.6 .

\section{THE CINCINNATI PHOSPHATE COMPANY,}

Cincinnati, OHio.

4797. CAPITOL GRAIN AND GRASS GROIIER. Shanklin Bros., Lowell. Guarantee: Phosphoric acid, soluble, 8.00 ; reverted, 2.00; insoluble, 1.00 ; total, 11.00 ; available. 10.00 ; nitrogen, 0.82 ; potash, 1.00 ; phosphoric acid from mineral phosphate, bone and animal tankage; nitrogen from tankage; potash from muriate and carbonate. Found: Phosphoric acit, soluble, 7.15 ; reverted, 5.00 ; insoluble, 1.09 ; total, 13.24 ; available, 12.15 ; nitrogen, 0.92 ; potash, 1.35 ; chlorine, 1.6 ; availability of nitrogen, 70 .

\section{IIARIETTA BONE AND PHOSPHATE COIPANY, Marietta, OHio.}

4673. MARIETTA. POTATO AND TRUCK SPECIAL. P. A. Johnson, Parkersburg. Guarantee: Phosphoric acid, total, 11.00 ; available, 8.00 ; nitrogen, 3.00 ; potash, 5.00 ; phosphoric acid from acid phosphate; nitrogen from nitrate of soda, tank“ge and bone; potash from sulphate of potash. Found: PhosYhoric acid, soluble, 2.19; reverted, 7.55; insoluble, 2.83; total. 12.67 ; available, 9.84 ; nitrogen, 1.56 ; potash, 4.66 ; chlorine, 0.6 ; availability of nitrogen, 86 . 
46Tt. WARIETTA HORSE BRAND CORN, OATS ANI) IIHEAT GROWER. R. A. Johnson, Parkersburg. Gua:untre: Total phosphoric acid, 11.00 ; available, 8.00 ; nitrogen, ".00; potash, 2.00: phosphoric acid from acid phosphate anci hone: nitrogen from bone and tankage; potash from sulphate of potash. F'ound: Phosphoric acid, soluble, 0.50; reverted, 8.26: insoluble, 3.51 ; total, 12.27 ; available, 8.76 ; nitrogen, 1.69; potash, 2.62; chlorine, 1.7 ; availability of nitrogen, 78 .

\section{MILLER FERTILIZER COMPANY,}

Baltimore, MD.

4703. GROUND BONE. J. L. Loughric, Rowlesburg. fuaruntec: Total phosphoric acid, 1t.00; nitrogen, 2.47 from animal bone. Found: Phosphoric acid, total, 16.34; nitroger, 3.02 ; availability of nitrogen, 75 .

4704. S. C. ROCK. J. L. Loughrie, Rowlesburg. Gu!ar(nntce: Phosphoric acid. soluble, 10.00 ; reverted, 4.00 ; insolubls, 1.5 : total, 15.50 : arailable, 14.00 ; from acid phosphate. Found: Thosphoric acid, soluble, 2.12 ; reverted, 4.62; insoluble, 0.70; total, 17.44 ; available, 16.74 .

4705. TOOLDRIDGE \& ANDERSON'S GERMAN ATIALINE MITURE. J. L. Loughrie, Rowlesburg. Gu(uanice. Phosphoric acid, soluble, 7.00 ; reverted, 3.00 ; insoluble. 1.00 ; total, 11.00 ; available, 10.00 ; potash, 2.00 ; phosphoric acid from acid phosphate; potash from manure salts. Found: Phosphoric acid, soluble, 2.12 ; reverted, 6.40 ; insoluble, $1.5 \mathrm{i}$; total, 10.03 ; available, 8.52 ; potash, 2.49 ; chlorine, 2.8 .

4706. WOOLDRIDGE of ANDERSON'S TIGER BONF STOCK PHOSPHATE. J. L. Loughrie, Rowleshurg. Guarantee: Phosphoric acid, soluble, 5.00; reverted, 3.00 ; insoluble, 1.50; total, 9.50; available, 8.00 ; nitrogen, 0.82 ; potash, 5.00 ; phosphoric acid from dissolved bone, tankage and acid phos- 
phate; nitrogen from dissolved bone and tankage, nitrate of soda ; potash from muriate of potash. Found: Phosphoric acid, soirbie, 3.68 ; reverted, 4.33; insoluble, 1.97; total, 9.98 ; available, 8.01 ; nitrogen, 0.78 ; potash, 4.63 ; chlorine, 1.6 ; availability of nitrogen, 84 .

4707. WOOLDRIDGE \& ANDERSON'S SPECIAL PO'I'ATO AND TOMATO GROWER. J. L. Longhrie, Rowlesburg. Guarantee: Phosphoric acid, soluble, 6.00 ; reverted, 3.00 ; insoluble, 1.50 ; total, 10.50 ; available, 9.00 ; nitrogen, 1.65 ; potash, 5.00 ; phosphoric acid from dissolved bone and tankage and acid phosphate; nitrogen from dissolved bone and tankage and nitrate of soda; potash from muriate of potash. Found: I'hosphoric acid, soluble, 6.49 ; reverted, 2.25; insoluble, 0.78; total, 9.52 ; available, 8.74 ; nitrogen, 1.65 ; potash, 4.53 ; chlorin , 1.0 ; availability of nitrogen, 89 .

4708. WOOLDRIDGE \& ANDERSON'S BATTLE-AXE IOTASH MIXTURE. J. L. Loughrie, Rowlesburg. Guarantee: Phosphoric acid, soluble, 9.00 ; reverted, 3.00, insoluble, 1.00 ; total, 13.00 ; available, 12.00 ; potash. 3.00 ; phosphoric acid from acid phosphate; potash from manure salts. Found: Phosphoric acid, soluble, 3.98 ; reverted, 8.40 ; insoluble, 0.95 ; tntal, 13.33 ; available, 12.38 ; potash, 2.82 ; chlorine, 2.0.

\section{BALTIMORE PULVERIZING COMPANY,}

Battimore, MD.

4733. SPECIAL SPRING MIXTURE. R. N. Stewart \& Son, Martinsburg. Unregistered. Found: Phosphoric acid soluble, 1.04; reverted 6.77 ; insoluble, 1.10 ; total, 9.91 ; available, 8.81; potash, 1.39 ; chlorine, 3.00 .

\section{G. OBER \& SONS COMPANY,}

Battimore, MD.

4685. OBER'S FARMERS MITTURES. A. G. Chrıs. 
lip, Philippi. Guarantec: Phosphoric aeid, soluble, 7.00 ; r: verted, 2.00 ; insoluble, 2.00 ; total, 11.00 ; available, 9.00 ; nitrog'u, (1.82; potash, 2.00; phosphoric acid from high grade Floricla phosphate, bone, tankage and fish: nitrogen from high grade bone and blood, tankage and fislı; potash from sulphate of potash. Found: Phosphoric aeid soluble, 7.37 ; reverted. 2.09; insoluble, 0.99 ; total, 10.45 ; available, 9.46 ; nitrogen, 0.89 ; potash, ¿.54; chlorine, trace; availability of nitrogen, 85 .

\section{OBER'S DISSOLVED BONE PHOSPHATE.} A. G. Chrislip, Philippi. Guarantee: Phosphorie acid, solnble, 11.00 ; reverted. 3.00 ; insoluble, 2.00 ; total, 16.00 ; available, 14.00 ; from high grade Florida phosphate. Founl: 'hosphoric acid, soluble. 16.53 ; reverted, 1.45 ; insoluble, 0.15; total, 18.13 ; available, 17.98 .

4732. OBER'S DISSOLTED BONE PHOSPHATE. R. N. Stewart \& Son, Martinsburg. Guarantee-(As above.) Found: Phosphorie acid, soluble, 10.57; reverted, 6.18; insoluble, 1.33 ; total, 18.06 ; available, 16.73 .

\section{PIEDMONT IIT. AIRY GUANO CONIPANY,}

Cincinnati, Ohio.

4727. PIEDMONT POTATO PRODUCER. C. Phillips, Belington. Guarantees Phosphorie aeid, insoluble, 2.00 ; total, 7.00 ; available, 5.00 ; nitrogen, 2.47 ; potash, 6.00 ; phosphoric acid from dissolved bone and phosphate rock; nitrogen from hone, blood, tankage and nitrate of soda; potash from hich grade muriate of potash. Found: Phosphoric acid, soluhle, 4.95 ; reverted, 1.80 ; insoluble, 0.65 ; total, 7.43 ; available, 6.78 ; nitrogen, 2.38 ; potash, 5.61 ; chlorine, 1.40 ; availability of nitrogen, 85.

472S. PIEDMONT FARUERS' HIGH GRADE BONE LND POTASH. C. Phillips, Belington. Guarantee: Phos- 
phoric acid, soluble, 2.00; reverted; total. 12.00; availalsh, 10.00 ; potash, 2.00; phosphoric acid from S. C. rock: potash fror.1 kainit. Found: Phosphoric acid, soluble, 7.94; reverted, 2.7\$; insoluble, 0.68 ; total, $11 . \frac{1}{1} 0$; available, 10.72 ; potash, 2.33; ch!orine, 3.2 .

\section{SWIFT \& COMPANT,}

\section{Chicago, Ill.}

4776. SWIFT'S CHAMPION WHEAT AND CORN GROTER. J. II. Miller \& Bro., Ronceverte. Guarante: phosphoric acid, insoluble, 1.00 ; total, 13.00 ; available, 12.00 ; nitrogen, 1.64; potash. 2.00; phosphoric acid from pure animal bone, acidulated; nitrogen from animal bone: potash from muriate. Fouvd: Phosphoric acid, soluble, 2.03: reverted, 11.27; insoluble, 2.18: total. 15.48; available, 13.30; nitrogen, 1.82; potasli, 2.00 ; chlorine, 0.9 ; availability of nitrogen, 93 .

4778. SWIFT'S POTATO AND TOBACCO GROTIEI;. J. Mr. Miller \& Bro., Ronceverte. Guarantee: Phosphoric acid, insoluble, 1,00; total, 11.00: available, 10.00; nitrogen, 3.25; potask, 5.00 ; phosphoric acid from acidulated rock and animal bone; nitrogen from bone, blood and tankage; potash from muriate of jotash. Found: Phosphoric acid, soluble, 7.14; reverted, 4.05; insoluble, 4.07 ; total, 15.26 : available, 11.19; nitrogen, 2.72 ; potash, 4.44; chlorine, 0.1 ; availability of nitrogen, 82.

4779. SWIFT'S SPECIAL BONE HEAL. J. MI. Milic! \& Bro., Ronceverte. Guarantee: Total phosphoric acid, 27.50, nitrogen, 0.82; from animal bone. Found: Phosphoric acicl, 31.32 ; nitrogen, 1.19 ; arailability of nitrogen, 89.

4781. SWIFT'S SUPERPHOSPHATE. J. MI. Miller \& Bro., Ronceverte. Guarantee: Phosphoric acid, insoluble, 4.jo); total, 12.00 ; available, 8.00 ; nitrogen, 1.64 ; potash, 2.00 ; phosphoric acid from animal bone and acidulated phosphate rocir; nitrogen from animal bone, blood and tankage; potash from 
muriate. Found: Phosphoric acid, soluble, 3.77 ; reverted, 3.92 ; msoluble, 2.26 ; total, 9.95 ; available, 7.69 ; nitrogen, 1.46 ; potash, 1.96 ; chlorine, 0.7 ; availability of nitrogen, 88 .

4734. SWIFT'S SUPERPHOSPHATE. R. N. Stewart \& Son, Martinsburg. Guarantee-(As above.) Found: Phosphoric acid, soluble, 3.59: reverted, 3.99; insoluble, 2.10; total, 9.68; available, 7.58 ; nitrogen, 1.53 ; potash, 2.17 ; chlorine, $0 . \mathbf{1}$; availability of nitrogen, 89 .

4735. SWIFT'S BONE MEAL. R. N. Stewart \& Son, Martinsburg. Guarantee: Total phosphoric acid, 25 ; nitrogen, 2.50; from animal bone. Found: Phosphoric acid, total, 27.47; nitrogen, 2.10 ; availability of nitrogen, 84 .

\section{VIRVINIA CAROLINA CHEMICAL COMPANY.} RICHMOND, VA.

4697. T. C. C. CO.'S GUARANTEED 14 PER CEVT' ICID PHOSPHATE. . . H. Phillips \& Son, Berkeley Springs. Gcods not registered. Found: Phosphoric acid, solnble, 11.37: reverted, 3.74 ; insoluble, 1.52 ; total, 16.63 ; available, 15.11 .

4791. ALLISOY \& ADDISON'S STANDARD ACID PHOSPHATE. E. W. ICNeer, Lowell. Guarantec: Pho=thoric acid, soluble, 9.00 ; reverted, 3.00 ; insoluble, 1.00; tota!, 13.00 ; available, 12.00 ; from phosphate rock. Found: Phosphoric acid, soluble, 5.04; reverted, 6.43; insoluble, 3.61 ; total, 15.08 ; available, 11.47 .

4798. ALLISON \& ADDISON'S LITTLE GIANT GRAIN IND GRASS GROWER. J. M. Niller \& Bro., Ronceverte. Guarantee: Phosphoric acid, soluble, 6.00 ; reverted, 2.00 ; insoluble, 2.00 ; total, 10.00 ; available, 8.00 ; nitrogen, 0.82 ; potash, 2.00; phosphoric acid from phosphate rock and animal bone; nitrogen from fish, animal, tankage and cotton-seed meal; potash from muriate, sulphate and double manure salts. Found: 
Phosphoric acid, soluble, 3.45; reverted, 4.43; insoluble, 2.34; total, 10.22 ; avajlable, 7.88 ; nitrogen, 0.92 ; potash, 2.24 ; chlorine, 1.9 ; availability of nitrogen, 74 .

4801. ALLISON \& ADDISON'S LITTLE GIANT GRAIN IND GRASS GROWER. E. W. McNeer, Lowell. Guarc'itee-(As above.) Found: Phosphoric acid, soluble, 4.71; reverted, 2.94; insoluble, 2.16 ; total, 9.81 ; available, 7.65 ; nitrogen, 0.80 ; potash, 2.31; chlorine, 2.9; availability of nitr $)$ w.n, 80 .

4799. ALLISON \& ADDISON'S B. P. POTASH MIX. IURE. E. W. McNeer, Lowell. Guarantee: Phosphoric acici, soiuble, 7 ; reverted, 3 ; insoluble, 1 ; total, 11 ; available, 10 ; po!ash, 2.00 ; phosphoric acid from phosphate rock; potash from muriate, sulphate and double manure salt. Found: Phosphoric acid, soluble, 3.17 ; reverted. 6.14 ; insoluble, 2.19 ; total, 11.59; available, 9.31 ; potash, 1.77 ; chlorine, 3.2.

4790. S. W. TRAVER'S DISSOLVED BONE PHOSPHATE. E. W. MeNeer, Lowell. Guarantec: Phosphoric acid, soluble, 11.00 ; reverted, 3.00 ; insoluble, 1.00 ; total, 15.00; available, 14.00; from phosphate rock. Found: Phosphoric, acid, soluble, 6.68 ; reverted, 7.06 ; insoluble, 2.93 ; total, 16.67 ; available, 13.74

4800. S. W. TRAVER'S CHAMPION CORN GROWER. E. W. MeNeer, Lowell. Guarantee: Phosphoric acid, soluble, i).00; reverted, 2.00 ; insoluble, 2.00 ; total 10.00; available, 800 ; nitrogen, 0.82 ; potash, 1.00 ; phosphoric acid from phosphati rock; nitrogen from fish, animal, tankage, and cotton-seed meal; potash from muriate, sulphate and double manure salts. Found: Phosphoric acid, soluble, 5.08; reverted, 4.93; insoluble, $\because .81$; total, 12.82 ; available, 10.01; nitrogen, 1.01 ; potash, 1.29 ; hlorine, 1.3 ; availability of nitrogen, 80 .

4789. S. W. TRAVER'S CAPITOL DISSOLVED S. C. 
BONE. E. W. MeNeer, Lowell. Guarantee: Phosphoric aeici, soluble, 9.00 ; reverted. 3.00 ; insoluble, 1.00 ; total, 13.00 ; avail. able, 12.00; from pliosphate rock. Found: Soluble, 7.43 ; revert('.l, 7.13 ; insoluble, 1.89 ; total, 16.45 ; available, 14.56.

4796. TINSLEY'S ACID PHOSPHATE. E. W. MeNeer, Lowell. Goods not registered. Found: Phosphoric acid, soluble, 6.06 ; reverted, 6.56 ; insoluble, 2.00; total, 14.62; available, 12.62 .

\section{WALKER STRATMANN \& COMPANY, Pittsburg, PA.}

4658. FOUR FOLD. Moore \& Hibbs, Mannington. Gun)'ntee: Phosphoric acid, soluble, 4.00 ; reverted, 4.00; insolubl?, 1.00 ; total, 9.00 ; available, 8.00 ; nitrogen, 1.50 ; potash, $2.0 c^{\prime}$; phosphoric acid, one-third from tankage, two-thirds from mirexal phosphates; nitrogen, from blood and tankage; potash from sulphate of potash. Found: Phosphoric acid, soluble, 4.81; reverted, 1.88 ; insoluble, 1.65 ; total, 8.24 ; available, 6.69 ; nitrogen, 1.95 ; potash, 2.43 ; ehlorine, 2.7 ; availability of nitrogen, 76 .

4670. FOUR FOLD. J. S. Liggett, Wellsburg. Gu'yuntee-(As above.) Found: Phosphoric acid, soluble, 3.99; reverted, 1.96; insoluble, 1.93 ; total, 7.88 ; available, 5.95 ; nitıoGen, 1.06 ; potash, 2.41; chlorine, 2.9; availability of nitrogin, 85 .

4749. FOUR FOLD. J. D. Owens, Weston. GuaranteeAs above.) Found: Phosphoric acid, soluble, 4.31; reverted, 2.16; insoluble, 1.80 ; total, 8.29; available, 6.49 ; nitrogen, 0.96 ; potash, 2.15 ; chlorine, 2.8 ; availability of nitrogen, 86 .

4751. FOTR FOLD. A. P. Russell \& Co., Buckhannon. Guruntce-(As above.) Found: Phosphoric acid, soluble, 4.29 ; reverted, 1.71 ; insoluble, 2.00 ; total, 2.00 ; total, 8.47 ; avaitahle, 6.00 nitrogen. 1.16 ; potash, 2.20 : chlorine, 2.7 ; availability of. nitrogen, 82. 
4659. POTATO SPECIAL. MIoore \& Hibbs, Mannington. Guarantee: Phosphoric acid, soluble, 4.00 ; reverted, 4.00 ; inso!uble, 2.00 ; total, 10.00 ; arailable, 8.00 ; nitrogen, 2.50 ; potash, b.00; phosphoric acid, two-fifths from tankage, three-fifths from mineral phosphates; nitrogen from tankage and blood; potash from muriate of potash. Found: Phosphoric acid, solubie, 3.61 ; reverted, 3.48 ; insoluble, 2.94; total, 10.03 ; available, 7.09 ; nitrogen, 2.53; potash, 5.86; chlorine. 0.9 ; availability of nitrogen, 89.

4668. POTATO SPECIAL. J. S. Liggett, Wellsburg. (ivarantee-(As above.) Found: Phosphoric acid, soluble, 4.29 ; reverted, 3.44 ; insoluble, 3.57 ; total, 11.30 ; available, 7.73 ; uitrogen, 2.69 ; potash, 4.35 ; chlorine, 1.3 ; availability of nitrogen, 88 .

4772. POTATO SPECIAL. Thomas Nuzum, Grafton. Gilarantee-(As above.) Found: Phosphoric acid, soluble, 3.35 ; reverted, 3.36 ; insoluble 3.04 ; total, 9.75 ; available, 6.71 ; nitrogen, 2.38 ; potash, 5.20; chlorine, 0.9 ; availability of nitrogen, 90 .

4660. HELP MATE. Moore \& Hibbs, Mannington. Guarantee: Phosphoric acid, soluble, 7.00; reverted, 5.00; insoluble, 1.00 ; total, 13.00 ; available, 12.00 ; from mineral phosphate. Found: Phosphoric acid, soluble, 5.59; reverted, 4.62; insoluble, 2.29; total, 12.50 ; available, 10.21 .

4669. HELP MATE. J. H. Liggett, Wellsburg. Guarantee-(As above.) Found: Phosphoric acid. soluble, 6.05; reverted, 5.93; insoluble, 2.35; total, 14.33; available, 11.98 .

4753. HELP MATE. J. D. Owens, Weston. Guarantee -(As above.) Found: Phosphoric acid, soluble, 6.00 ; revertel, 4.13: insoluble, 2.69; total, 12.82; available, 10.13.

4667. GRAIN KING. J. S. Liggett, Wellsburg. Guarantee: Phosphoric acid, soluble, 5.00 ; reverted, 4.00 ; insoluble, 1.00 ; total, 10.00 ; available, 9.00 ; nitrogen, 0.82 ; potash, 4.00; 
1, Josphoric acid, one-fifth from tankage; four-fifths from minerai phosphate; nitrogen from tankage and blood; potash from muriate of potash. Found: Phosphoric acid, soluble, 4.48; r... rerted, 2.42; insoluble, 2.23; total, 9.13 ; available, 6.90 ; nitrogen, 1.10 ; potash, 1.99 ; chlorine, 21; availability of nitrogen, 8..

4754. GRAIN KING. J. D. Owens, Weston. Guaranle: : - (As above.) Found: Phosphoric acid, soluble, 5.14 ; revertel, 1.96 ; insoluble, 1.36 ; total, 8.46 ; available, 7.10 ; nitrogen, 0.87 ; iotash, 1.63; chlorine, 2.4 ; availability of nitrogen, 68 .

4755. GRAIN KING. A. P. Russell \& Co., Buckhannon. Guarantee-(As above.) Found: Phosphoric acid, soluble, 3.26 ; reverted, 1.72 ; insoluble, 1.41 ; total, 8.39 ; available, 6.98 ; nitrogen, 1.10 ; potash, 1.62 ; chlorine, 2.4 ; availability of nitrogen, 78 .

4671. GRAIN MANURE. J. S. Liggett, Wellsbrug. Guarantee: Phosphorie acid, soluble, 4.00; reverted, 4.00; insoiuble, 1.00 ; total, 9.00 ; available, 8.00 ; nitrogen, 0.82 ; potasin. 1.00 ; phosphorie acid, one-fiftl from tankage; four-fifths frou mineral phosphate; nitrogen from tankage and blood; potasin lrom sulphate of potash. Found: Phosphoric acid, soluble, 4.58 ; reverted, 1.93 ; insoluble, 1.93 ; total, 8.44 ; available, 6.51 ; nitrogen, 0.93 ; potash 1, 1 . 45 ; chlorine, 2.6 ; availability of nitrogen, 79 .

4672. PURE RAW BONE MEAL. J. S. Liggett, Wellsbürg. Guarantee: 'Total phoshoric aeid, 22.00 ; nitrogen, 3.25 ; from hone. Found: Total phosphoric acid, 21.16; nitrogen, 3.21; availability of nitrogen, 87 .

4750. BIG BONANZA. A. P. Russel \& Co., Buckhannon. Ginarantee: Phosphoric acid, soluble, 4.05; reverted, 4.05; insoluble, 1.00 ; total, 10.00 ; available, 9.00 ; nitrogen, 1.50 ; potash, 1.00; phosphorie acid, one-third from tankage, two-thirds from nineral phosphates; nitrogen from blood and tankage; potasiı 
from muriate of potash. Found: Phosphoric acid, soluble, 3.90; reverted, 3.03 ; insoluble, 2.05 ; total, 9.07 ; available, 7.02 ; nitrogen, 1.69 ; potash, 3.91 ; chlorine, 1.0 ; availability of nitrogen, 87.

4752. PHOSPHORIC ACID AND POTASH. J. D. ()wens, Weston. Guarantee: Phosphoric acid, soluble, 5.50; reverted, 4.50 ; insoluble, 1 ; total, 11 ; available, 10 ; potash, 2 ; phosphate from mineral phosphate; potash from sulphate of potash. Fcund: Phosphoric acid, soluble, 5.21; reverted, 3.23 ; insoluble, 1.92 ; total, 10.36 ; available, 8.44 ; potash, 1.87 ; chlorine, 3.1.

\section{ROB'. A. WOOLDRIDGE COMPANY,}

\section{Baltimore, Md.}

4717. FLORIDA ACID PHOSPHATE. Griffith \& Howcil, Belington. Guarantee: Phosphoric acid, available, 14.00. from dissolved phophate rock. Found: Phosphoric acid, solnble, 11.71; reverted, 3.87 ; insoluble, 1.05 ; total, 16.63 ; available, 15.58 .

4725. GERMAN POTASH MIXTURE. Griffith \& Howeli, Belington. Guarantee: Phosphoric acid, available, 10.00 ; potash, 2.00; phosphoric acid, from dissolved phosphate rock; petash from German kainit. Found: Phosphoric acid, solubje, 7.46; reverted, 4.36; insoluble, 0.76 ; total, 12.58 ; available, 11.82 ; potash, 2.16 ; chlorine, 4.3 .

4726. IIBERTY BELL POTASH MIXTURE. Griffit in \& Howell, Belington. Guarantee: Phosphoric acid, available, 12.00; potash, 3.00; phosphoric acid, from disolved phosphate rock; potash from kainit and manure salts. Found: Phosphoric acid, soluble, 9.81 ; reverted, 2.19 ; insoluble, 0.46 ; total, 12.36; available, 11.90 ; potash, 3.09 ; chlorine, 3.0 . 
IBRANISS FOUND DEFICIEN'T OR CONTLARY TO GUARANTEE.

The results of the analyses of fertilizers found on sale in the State show the following brands to have fallen short or to be contrary to guarantee, as stated blow:

4786. CANTON CHEMICAL SOL. BONE AND POTISII. Guarantee: Potash, 2.00. Found: 1.71.

4701. CANTON CHEMICAL POTATO MANURE. (inarantec: Potash, 5.00. Found: 4.67

4785. CANTON CHEMICAL POTATO MANURE. Guarantec: Potash, 5.00. Found: 4.86.

4699. CANTON CHEMTCAL SOLUBLE ALKALINE BONE. Guarantee: Potash, 3.00. Found: 2.67.

4765. CLEVELAND DRYER FOREST CITY BUCíLIE. Gutrantee: Available, 9.00. Found: 8.58. Guaantec: Nitrogen, 2.47. Found: 1.92

4724. LAZARETTO HIGH GRADE DIS. BONE 'HOSPHATE AND POTASH. Guarantee: Potash, 5.00. Found: 4.70.

4762. LAZARETTO SPECIAL POTATO FERTILIZEP. Guarantee: Available, 8.00. Found: 7.19 .

4748. BAUGH'S CORN FERTILIZER. Guarantee: Potash, 4.00. Found: 2.38 .

4747. BAUGH'S SPECIAL POTATO MANURE. Guarmutce: Potash, 10.00. Found: 7.39 Guarantce: Sulphate, muriate and kainit. Found: Muriate.

4757. BOWKER'S HARVEST BONE. Guarantec: Available, 8.00. Found: 7.41 . 
COMMERCLL FERTILIZEIS - REPORT FOR 1903.

4794. BOWKERS DIS. BONE AND POTASH. Guaranteed: Muriate. Found: Kainit.

4673. MARIETTA POTATO AND TRUCK SPECIAL. Ginarantee: Nitrogen, 3.00. Found: 1.56. Guarantee: Potash, 5.00. Found: 4.66 .

4674. MARIETTA CORN, OATS AND WHEAT GROWER. Guarantee: Nitrogen, 2.00. Found: 1.69. Guarantec: Sulphate of potash. Found: Muriate.

4705. MILLER'S W. AND A. GERMAN ALKALINE MIXTURE. Guarantee: Available, 10.00. F'ound, 8.52.

4706. MILLER'S W. AND A. TIGER BONE STOCK PHOSPHATE. Guarantee: Potash, 5.00. Found: 4.63.

4707. MILLER'S W. AND A. SPECIAL POTATO AND TOMATO GROWER. Guarantee: Potash, 5.00. Found: 4.53.

4733. B.ALTIMORE PULVERIZING CO., SPECIAL NDRING MIXTURE. (Not registered.)

4727. PIEDHONT POTATO PRODUCER. Guarantee: Potash, 6.00. Found: 5.61.

4778. SWIFT'S POTATO AND TOBACCO GROWER. (rilarantee: Nitrogen, 3.25. Found: 2.72. Guarantee: Potash, 5.00. Found: 4.44. Old tags.

4781. SWIFT'S SUPERPHOSPHATE. Guarantee: Nitrogen, 1.64. Found: 1.46. Guarantee: Available, 8.00. Found: 7.69 .

4734. SWIFT'S SUPERPHOSPHATE. Guarantee: Nitrogen, 1.64. Found: 1.53. Guarantee: Available, 8.0n. Found: 758 .

4735. SWIFT'S BONE MEAL. Guarantee: Nitrogen, 2.50. Found: 2.10 . 
4791. ILLISON \& ADDISON'S STANDARD AC!D FIIOSPIIATE. Guarantecd: Available, 12.00. Found: 11.千7.

4799. ALTISON \& ADDISON'S B. P. POTASH MLYTCRE. Guem?lice: Availab!e, 10.00. Found,9.31. Guar(titrc: Potash, 2.00. Found: 1.77. Guarantee: IIuriate, sulfhate and double manure salt. Foiins: Kainit.

4796. TINSLEY'S ACID PHOSPHATE. (T. C. C. CO.) Not registered nor tagged.

WALKER, STRATMAN \& COMPANY'S GOODS.

4658. FOUR FOLD. Guarantee: Arailable, 8.00 . Found: 6.69. Guarantee: Sulphate of potash. Found: Kainit.

4670. FOUR FOLD. Guarantee: Available, 8.00. F'ound: 5.95. Guarantec: Nitrogen, 1.50. Found: 1.06. Found: Kainit.

4749. FOUR FOLD. Guarantce: Available, 8.00 . Fcund: 6.49. Guarantec: Nitrogen, 1.50. Found: 0.96. Found: Kainit.

4751. FOUR FOLD. Guarantee: Available. 8.00 . Found: G.0n. Gualuntec: Nitrogen, 1.50. Found: 1.16. Found: Kainit, or low grade muriate.

4659. POTATO SPECIAL. Guarantee: Available, 8.00. Found: 7.09 .

4668. POTATO SPECIAL. Guarantee: Available, S.00. Found: 7.73. Guarantec: Potash, 6.00. Found: 4.35.

47i2. POTATO SPECIAL. Guarantee: Available, s.00. found: 6.71. Guarantce: Potash, 6.00. Found: 5.20.

4660. HELP MATE. Guarantee: Available, 12.00. Fonnd: 10.21 . 
4753. HELP MATE. Guarantee: Available, 12.00. Found: 10.13.

4667. GRAIN KING. Guarantee: Available, 9.00. Found: 6.90. Guarantee: Potash, 4.00. Found: 1.99. From low grade muriate.

4754. GRAIN KING. Guaranteed: Available, 9.00. i'oinä: 7.10. Guarantee: Potash, 4.00. Found: 1.6i3. From low grade muriate.

4755. GRAIN KING. Guarantee: Available, 9.00 Found: 6.98. Guarantee: Potash, 4.00. Found: 1.62. From low grade muriate.

4671. GRAIN MANURE. Guarantee: Available, 8.00. Found: 6.51. Guarantee: Sulphate of potash. Found: Low grade muriate.

4672. PURE RAW BONE MEAL. Guarantee: Total, 22.00. Found: 21.16.

4750. BIG BONANZA. Guarantee: Available, 9.01). Found: 7.02 .

4752. PHOSPHORIC ACID AND POTASH. Guarantee: Available, 10.00. Found: 8.44. Guarantee: Sulphate of potash. Found: Kainit.

*4688. ARMOUR'S HIGH GRADE POTATO. Guaruniee: Muriate and sulphate. Found: Low grade sulphate.

*4687. ARMOUR'S WHEAT, CORN AND OATS SPECIAL. Guarantee: Muriate, sulphate and kainit. Found: low grade muriat.

* Guarantees concerning sources of potash not specific. In the future, affidavits, to be accepted, must be fully explicit in all required details. 
*4690. ARMOUR'S GRAIN GROWER. Guaranter: Muriate, sulphate and kainit. Found: Kainit.

*4798. ALLISON \& ADDISON LITTLE GIANT GRAIN AND GRASS GROWER. Guarantee: Mruriate, sulphate, an! double manure salts. Found: Isow grade muriate.

*4801. ALLISON \& ADDISON LITTLE GIANT GRAIN AND GRASS GROWER. Guarantee: Muriate, sulphate and (iouble manure salts. Found: Low grade muriate.

*4800. S. W. TRAVERS' CHAMPION CORN GROWER. Guarantee: Muriate. sulphate and double manure salts. Found: Muriate.

4712. BRADLEY'S BEAN AND POTATO. (Not tagged.)

4674. DETRICK'S SOL. BONE AND POTASH. (Not. tagged.)

4792. A. A. C. CO.'S REGULAR CORN MIYTURE. (Not tagged.)

Resides the above a number of brands are deficient 0.1 per cent or less in potash. In very many eases, espeeially on A. 1 . Co.'s goods, the exeessive amount of chlorine present indieates the use of an inferior grade of kainit. where kainit is guaranfeed the chlorine may not exeed 2.8 per cent up to 4.5 per cent. This is frequently much exeeded.

*Guarantees concerning sources of potasn not specific. In the future, affidavits, to be accepted, must be fully explicit in all required detalls. 


\section{REGISTERED BRANDS.}

FOR 1903.

The following is a list of the brands which were recistered as required by law, up to date of Sept. 1st, 1903 :

\section{AMERICAN AGRICULTURAL CHEMICAL COMPANY.}

B.iltimore Sales Departaent.

Superphosphate.

Gem Alkaline Bone.

Regular Corn Fertilizer.

Dissolved Animal Bane.

Bone Meal.

Fine Ground Bone.

Pure Ground Bone.

W. \& A'. Special Fall Mixture.

Kainit.

Orchilla Guano.

Ruth's Dissolved Bone Phosphate.

Reliable.

Bradley's Alkaline Bone.

Bradley's Dissolved Bone with Potash.

Bradley's Bean and Potato Potash.

Bradley's Niagara Phosphate.

Bradley's Alkaline Bone.

Canton Chemical Eagle Phosphate.

Canton Chemical Potato Manure.

Canton Chemical Resurgam Guano.

Canton Chemical Game Guano.

Canton Chemical C. C. C. Special Compound.

Canton Chemical Special Wheat, Corn and Grass Fertilizer.

Canton Chemical "Harrow" Brand Crop Grower.

Canton Chemical Soluble Bone and Potash. 
Canton Chemical Dissolved S. C. Bone.

Canton Chemical Solnble Alkaline Bone.

Canton Chemical Patron's Bone Mixture.

Cleveland Dryer Forest City Buckeye.

Cleveland Dryer Phospho Bone.

Cleveland Dryer Horse Head Phosphate.

Cleveland Dryer Horse Head Phosphate with Potash.

Clcveland Dryer XXX Phosphate.

Detrick Quick Step Bone Phosphate for Potatoes and 'Tr. bacco.

Detrick Soluble Bone Phosphate and Potash.

Detrick Kangaroo Komplete Kompound.

Detrick Dissolved S. C. Bone.

Detrick Standard Potash Fertilizer.

Detrick Corn and Oats Fertilizer.

Detrick Imperial Compound.

Detrick Paragon Ammoniated Dissolved Bone Phospliate and Potash.

Tazaretto Bone Compound.

Lazaretto Special Potato Fertilizer.

Iazaretto Ammoniated Bone Phosphate.

Lazaretto Excelsior A. A. A.

Lazaretto Dissolved Bone Phosphate and Potash.

Lazaretto High Grade Dissolved Bone and Potash.

Lazaretto Dissolved Bone Phosphate.

Maryland Ammoniated Bone.

Maryland O. K. Ammoniated Fertilizer.

Maryland Tornado Fertilizer.

Maryland Bono Super-phosphate.

Maryland Dissolved S. C. Plosphate.

Pacific Nobsque Guano.

Pacific A. No. 1 Phosphate.

Pacific Potato Phosphate.

Pacific Dissolved Bone and Potash.

I'acitic Dissolved Bone Phosphate. 
Reese's Half and Half.

Reese's Elm Phosphate.

Reese's 'High Grade Potash Mixture.

Susquehannah Alkaline Bone Phosphate.

Susquehannah Potato Phosphate.

Susquehannah XXV Phosphate.

Susquehannah Superior Rock Phosphate.

Williams' \& Clark's Royal Bone Phosphate.

Williams' \& Clark's Prolific Crop Producer.

Williams' \& Clark's Good Grower Potato Phosphate.

Williams' \& Clark's Dissolved Bone and Potash.

Williams' \& Clark's Acorn Acid Phosphate.

Zell's Special Compound for Potatoes and Vegetables.

Zell's Ammouniated Bone Super-phosphate.

Zell's Eeonomizer Phosphate.

Zell's Little Giant.

Zell's Electric Phosphate.

Zell's Dissolved Bone Phosphate.

Zell's Dissolved S. C. Phosphate.

Great Eastern Unammoniated Wheat Special.

Great Eastern Corn Fertilizer.

Great Eastern Vegetable, Vine and Tobaceo.

Great Eastern English Wheat Grower.

Great Eastern Soluble Bone and Potash.

Great Eastern Dissolved Bone.

Great Eastern Wheat Special.

M. E. Wheeler \& Co.'s Corn Fertilizer.

M. E. Wheeler \& Co.'s Potato Manure.

M. E. Wheeler \& Co.'s Royal Wheat Grower.

M. E. Wheeler \& Co.'s Wheat and Clover Fertilizer.

M. E. Wheeler \& Co.'s Electrical Dissolved Bone.

M. E. Wheeler \& Co.'s Unammoniated Wheat Grower.

THE ABBOTT \& MARTIN RENDERING COMPANY, Columbus, OHio.

Ideal Grain Grower. 
Tennessee Phosphate.

Hercules Phosphate.

Star Phosphate.

Peerless Bone and Potash.

Harvest King.

Abbott's Potash and 'Tobateco Special.

Fine Raw Bone Meal.

\section{ARMOUR FERTILIZER WORKS, Baltimore, MD.}

Star Phosphate.

Phosphate and Potash No. 1.

Ammoniated Bone with Potash.

High Grade Potato.

Grain Grower.

Wheat, Corn and Oats Speeial.

Cereal Phosphate.

Bone Meal.

Wheat Special.

Acid Phosphate.

THE BALTIMORE PULVERIZING COMPANY, Baltimore, MD.

Special Fall Mixture.

Farmer's Favorite Fertilizer.

S. C. Bone.

BAUGH \& SONS COMPANY.

Baltimore, Md.

Baugh's Corn Fertilizer.

Baugh's Potato Fertilizer.

Baugh's Fish Mixture.

Bangh's Special Potato Mamme. 
Baugh's Raw Bone Super-phosphate.

Baugh's Peninsula Grain Producer.

Baugh's Export Bone with Potash.

Baugh's Warranted Pure Bone Meal.

Baugh's Dissolved Animal Bones.

Baugh's Double Eagle Phosphate.

Baugh's General Crop Grower.

Baugh's Animal Bone and Potash Compound.

Baugh's Wheat Fertilizer.

Baugh's Soluble Alkaline Super-phosphate.

Kaugh's Soluble Alkaline Super-phosphate.

Baugh's High Grade Acid Phosphate or Dissolved S. C. Roci.

BOWKER FERTILIZER COMPANY.

Boston, Mass.

Bowker's Harvest Bone.

Bowker's Dissolved Bone with Potash.

Bowker's Soluble Bone.

Bowker's Bone Meal.

Bowker's Potash Fertilizer.

CHICAGO FERTILIZER COMPANY,

Chicago, Ill.

Bone, Blood and Potash.

Wheat and Corn Special.

No. 1 Acid Phosphate.

Diamond Phosphate.

Calumet Phosphate.

Western Bone Black and Potash.

Potato, Truck and Tobacco Fertilizer.

Chicago Raw Bone. 
CINCINNATI PHOSPHATE COMPANY, Cincinnati, Ohio.

Capitol Wheat Grower.

Capitol Grain and Grass Grower.

Capitol Dissolved Bone and Potash.

Capitol Ground Bone.

Capitol T'obacco Food.

GRIFFITH \& BOYD.

BultiaroRe, MD.

Griffitl's \& Boyd's Original Super-phosphate. Griffith's \& Boyd's Pure Fine Ground Bone Meal. Fruit Tree Fertilizer.

Stable Mamure Fertilizer.

Farmer's Potato Manure.

Spring Crop Grower.

Ammoniated Bone Phosphate.

Harvest Queen Phosphate.

Fish, Bone and Potash.

Cereal Bone Plant Food.

Valley Fertilizer.

Soft Ground Bone Meal.

Soft Ground Bone Meal.

Griffith \& Boyd's Grain and Grass Producer.

XX Potash Manure.

Special Grain Grower.

Peerless Fertilizer.

Double Strength Tobacco Grower.

High Grade Acid Phosphate.

Pure Dissolved Animal Bone.

Royal Potash Guano.

Farmer's Jupproved Phosphate. 


\section{JARECHI CHENICAL CONPANY, SANDUSKY, OHIO.}

No. 1 Fish Guano.

C. O. D. Phosphate.

Ground Bone.

Dissolved Bone with Potash.

Fish and Potash, Potato and Tobacco Food.

Phosphate and Potash.

MARIETTA BONE AND PHOSPHATE CONIPANY, Miarietta, OHio.

"Horse" Brand Pure Raw Bone.

"Horse" Brand Potato and Truck Special.

"Horse" Brand Corn, Oats and Wheat Grower.

"Horse" Brand Acid Phosphate.

"Horse" Brand Pure Ground Bone.

"Horse" Brand XX Special.

\section{B. MIARTIN COMPANY,}

PHILADELPHIA.

Dissolved Organic Compound.

Sure Grower.

Crop Producer.

Corn and Oats Special.

Claremont Vegetable Grower.

Steamed Bone.

Acid Phosphate.

Extra Bone and Potash.

\section{MILLER FERTILIZER COMPANY,}

BALTimore, MD.

Wooldridge \& Anderson's Old Hickory Phosphate.

Wooldridge \& Anderson's Faultless Ammoniated Bone

Phosphate.

Wooldridge \& Andersson's Tiger Bone Stock Phosphate. 
Wooldridge \& Anderson's Special Potato and Tomato Grower.

Wooldridge \& Anderson's 'F. F. V.' (Flesh and Fish Veg'tator.)

Wooldridge \& Anderson's Battle Axe Potash Mixture.

Wooldridge \& Anderson's German Alkaline Mixture.

Hustler Phosphate.

S. C. Rock.

W. G. Phosphate.

Special Tobacco Grower.

Standard Phosphate.

Ground Bone.

G. OBER \& SONS COMPANT,

Baltimore, MD.

Ober's Dissolved Animal Bone.

Ober's Farmer's Standard Ammoniated Phosphate.

Ober's Farmer's Mixture.

Ober's Independent Ammoniated Super-phosphate.

Ober's Dissolved Bone Phosphate.

Ober's Dissolved Bone Phosphate and Potash.

Ober's High Grade Acid Phosphate.

Ober's Pure Raw Bone Meal.

Ober's Special Mixture for Wheat.

Ober's S. \& S. Special Wheat Compound.

Ober's Shenandoah Bone Phosphate.

Langdon's Mixture.

OHIO FARMERS' FERTILIZFR COMPANY,

Columbus, Оніо.

Raw Bone Meal.

Com, Oats and Wheat Fish Guano.

General Crop Fish Guano. 
Wheat Maker and Seeding Down.

Potato and Tobacco Special.

Superior Phosphate.

Soluble Bone and Potash.

Alkaline Bone.

\section{PIEDMONT MT. AIRY GUANO COMPANY,} BALtimore, MD.

Piedmont Raw Bone Mixture.

Piedmont Royal Ammoniated Bone and Potash.

Piedmont Dissolved Bone Phosphate, Potash Goods.

Piedmont High Grade S. C. Bone.

Piedmont Potato Producer.

Piedmont "Plow" Brand.

Piedmont Special Potash Mixture.

Piedmont Soluble Bone and Potash.

Piedmont Harvest Queen.

Piedmont Farmer's Favorite.

Levering's Excelsior.

Magic Wheat Grower.

\section{POWHATAN CHEMICAL COMPANY, RICHMOND, VIRGINIA.}

Magic Special Fertilizer.

Magic Ammoniated Phosphate.

Magic Bone and Potash Mixture.

Bone and Potash Mixture.

Virginia Dissolved Bone.

High Grade Acid Phosphate.

RASIN MONUMENTAT, COMPANY, Bhltimore, Mo.

Rasin's XXX Fertilizer.

Rasin's Empire Guano.

Rasin's Bone and Potash. 
Rasin's Acid Phosphate.

Rasin's Dissolved Bone.

William Penn Crop Grower.

\section{RICHMOND GUANO COMPANY, Richiond, Virginia.}

"Premium" Brand Fertilizer.

"Premium" Brand Wheat Grower.

Bone Mixture.

Bone and Potash Mixture.

High Grade Acid Phosphate.

Dissolved S. C. Phosphate.

\section{SCIENTIFIC FERTILIZER COMPANY, Pittsburg, Penn.}

Scientific Phosphate and Potash.

Scientific Dissolved Bone.

Scientific Wheat and Clover.

Scientific Bone, Meat and Potash.

Scientific Bone and Potash.

Scientific Economy.

Scientific Potato.

Scientific Corn and Grain.

Scientific Grain Grower.

Bone and Meat.

Pure Raw Bone Meal.

High Grade Acid Phosphate.

\section{SWIFT \& COMPANY,}

Chicago, Ill.

Swift's Superphosphate.

Swift's Complete Fertilizer. 
Swift's Special Bone Meal.

Swift's Pure Raw Bone Meal.

Swift's Champion Wheat Grower.

Swift's Potato and Tobacco Grower.

Swift's Dissolved Animal Bone.

Swift's Bone Meal and Blood.

\section{P. THOMAS \& COMPANY,}

Philadel.phia, Penn.

S. C. Phosphate.

Alkaline Bone.

TUSCARORA FERTILIZER COMPANY, BALTiMORE, MD.

Acid Phosphate.

Bone and Potash.

Ammoniated Phosphate.

Special Crop Grower.

'Tuscarora Standard.

VIRGINIA-CAROLINA CHEMICAL, COMPANY, Richmond, Virginia.

Allison \& Addison Star Brand Guano.

Allison \& Addison Little Giant Grain and Grass Grower.

Allison \& Addison Standard Acid Phosphate.

Allison \& Addison B. P. Potash Mixture.

J. C. Tinsley's Bone and Potash Mixture.

S. W. Travers' Wheat and Grass Fertilizer.

S. W. Travers' Beef, Blood and Bone Fertilizer.

S. W. Travers' Champion Corn Grower Fertilizer.

S. W. Travers' Capitol Bone-Potash Compound.

S. W. Travers' Capitol Dissolved S. C. Bone.

S. W. Travers' Dissolved Bone Phosphate.

Standard Bone and Potash.

Syecial Compound for Wheat. 


\section{WALKER, STRATMAN \& COMPANT. Pittsburg, Penn.}

Phosphoric Acid and Potash.

Help Mate.

Pure Raw Bone Meal.

Bone and Neat.

Grain King.

Acid Phosphate.

Meat, Blood and Bone with Potash.

Potash and Bone Phosphate.

Big Bonanza.

Potato Special.

Four Fold.

Grain Manure.

\section{ROBERT A. WOOLDRIDGE COMPANY,} Baltimore, MID.

Florida Acid Phosphate.

German Potash Mixture.

Liberty Bell Potash Mixture.

Champion Giant Phosphate.

Chieftain Bone Stock Phosphate.

Triumph Bone Stock Phosphate.

Special Potato Fertilizer.

Pure Raw Bone.

LIST OF AGENTS

Whose Stock was SaMpled During 1903.

Armstrong, Chrislip \& Co., .............. Buckhanon (!. II. Becker . . . . . . . . . . . . . . . . . Wheeling Bishop \& Barbe ..................... Jane Lew W. H. Brady ................. Berkeley Springs R. P. Polyard, ..................... Fetterman W. H. Bailey Hardware Co.. . . . . . . . . . Morgantown

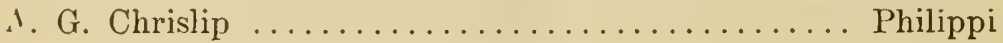


De Berry \& Bishop

Terra Alta

Drummond \& Co.,

Buckhannon

Ciriffith \& Howell

Belington

IIarrisville Mill Co.

Harrisvilie

.T. M. Hagerty

Farmington

F. Hunter

Berkeley Springs

Johnson \& Gwinn

Alderson

Ii. A. Johnson

Parkersburign

J. S. Liggett

Wellsburr

J. E. Loudermilk

Ronceverte

R. T. Lowndes

Clarksbur:

.J. S. Loughrie

Rowlesburg

L. P. Licklider

Martinsburg

J. M. Miller \& Bro.,

Ronceverte

.T. R. Morris

Gath

Yoore \& Hibbs

Manningteu

Ł. W. McNeer Lowe?!

'i homas Nuzum

Grafton

()ffut \& Lakin

Terra Alta

J. D. Owens

Weston

I'hilippi Mill Co.,

Philippi

C. Phillips' Sons

Belington

J. H. Phillips' Sons

Berkeley Springs

A. P. Russell \& Co.

Buckhannon

IV. F. Sill

Pennsboro

R. N. Stewrart \& Son

Martinsburg

( W. Siever

Keyser

Shanklin Bros.

Lowell

Washington \& Alexander

Charles Town

J. L. Woodyard

Pruntytown 
WEST VIRGINIA EXPERIMENT STATION..

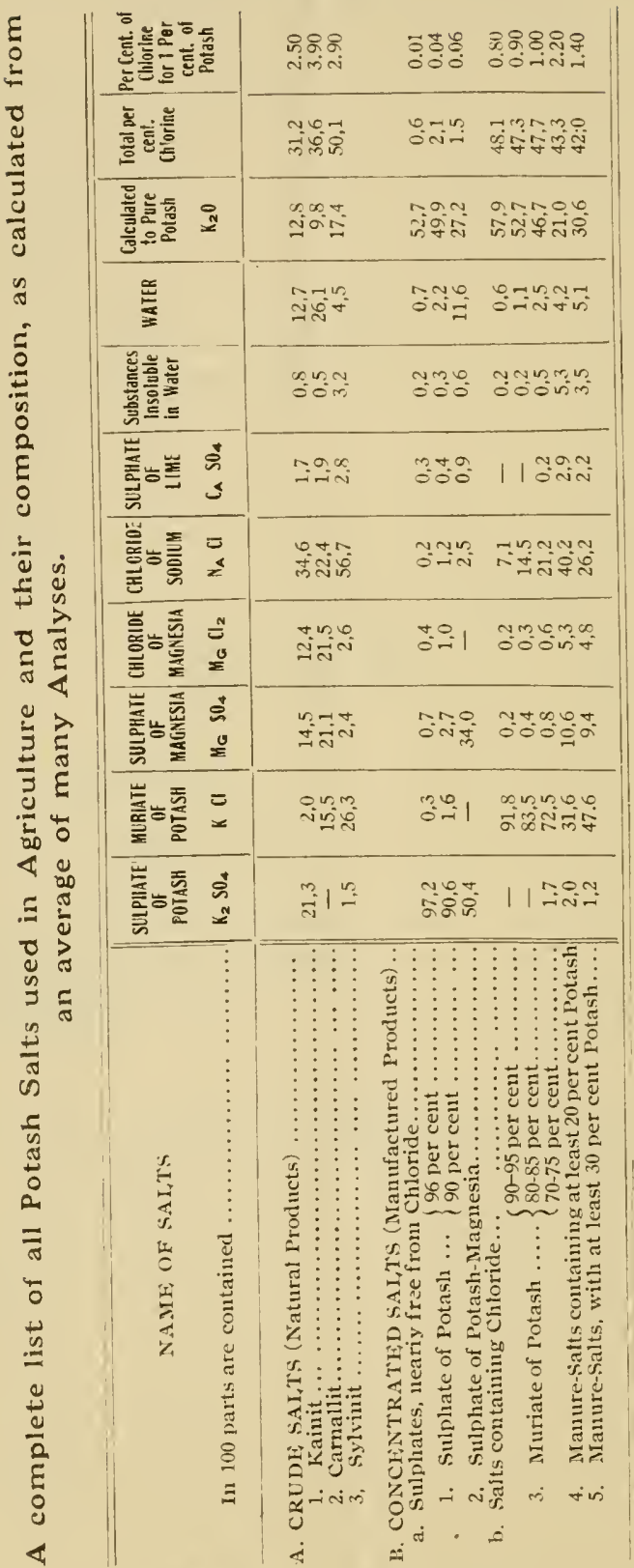




\section{AN ACT}

\section{Concerning Commercial Fertilizers, State of West Virginia.}

IN ACT concerning commercial fertilizers, and repealing chapter seventy-two of the Acts of the Legislature of West Virginia, of eighteen hundred and ninety-one.

[Passed February 2d, 1901.]

Be it enacted by the Legislature of West Virginia:

1. Every person who shall sell, offer or expose for sale, or have in his possession with intent to sell in this State, any cominercial fertilizer or manure, shall affix conspicuously to every fackage thereof a plainly printed statement, clearly and truly certifying the number of net pounds of fertilizer in a packagi, the name, brand or trade-mark under which the fertilizer is solr, the name and address of the manufacturer, the place of manuacture, and stating the percentage of nitrogen or its equivalent in ammonia: of potash, soluble in distilled water; and of phosr.horic acid in available form, soluble in distilled water, reverterl, as well as the total phosphoric acid, and the materials froin which said constituents are derived. In the case of those fertilizers which consist of other cheaper materials, said labels shail rive correct general statement of the composition and ingredients of the elements relied upon, contained in the fertilizer which it accompanies.

2. Before any commercial fetilizer is sold, offered or $\in \mathrm{x}$ posed for sale in this State, the manufacturer, importer or party who causes it to be sold, exposed or offered for sale, shall file with the Director of the West Virginia Agricultural Experiment Station, under oath, the statement required to be affixed undir section one of this act.

Provided, That when the manufacturer of any brand of fertilizer or manure shall file said sworn statement no agent or dealer shall be required to file such statement. The making of and affidavit required by this chapter falsely shall be perjury.

3. The manufacturer, importer. agent or seller of any brand of commercial fertilizer or material nsed for manurial purposes, shall pay for each brand at the time he files a sworn statement required by section two of this act a registration fee of one dollar per annum. 
4. The registration fee required to be paid by section three of this act, shall be paid to the treasurer of the West Virginia Lniversity for the use of the Agricultural Experiment Station. and the party making such parment shall take from the said treasurer triplicate reeeipts therefor, one of which he shall retain, and the other shall be deposited, one with the Director of the Agricultural Experiment Station, and the other with the secretary of the Board of Regents, and by them filed and pro. served in their respeetive offices.

5. Immediately after the filing of the receipts aforesair, with the Director of the Agrieultural Experiment Station, said Director shall issue a certifieate to the party making such payulent, stating the amount of fees paid, and the name, brand or trade-mark under which the fertilizer is sold, the name and address of the manufacturer or importer, the place of manufacture, and that the applicant for said certificate is authorizci to sell said fertilizer within the State of Test Virginia for the ieriod of one year from the first dayi of January to the thirtyfirst day of Deember, inclusire. Said certificates may be issued at any time for and during the current year, and may be issued during the month of December for the year commencing on the first day of January thereafter.

6. It shall be the duty of the Director of the West Virrinia Agricultural Experiment Station to print or cause to be printed a label or tag setting forth the sworn statement required in section two of this act. The Director shall furnish such labcls or tags in quantities in one hundred or multiples thereof, to any person complying with the requirements of this act, and desiring to sell. offer or expose for sale any commercial fertilizer iu this State, and shall receive pay therefor at the rate of forty cents for such number of labels or tags as may be required to le affixed to one ton of fertilizer, and shall. without delay, pay the same to the treasurer of the West Tirginia Unirersity, for use of the Agricultural Experiment Station, and take duplicate receipts therefor, one of which he shall retain, and the other he shall deliver to the seeretary of the Board of Regents, win. shall file and preserve the same in his office.

7. The Board of Regents of the West Virginia University thall expend the moner received under the provision of this act in meeting the legitimate expenses of the Station in making anajyses of fertilizers, in experimental tests of same, and in such other experimental work and purchases as shall inure to the henefit of the farmers of this State, and shall include in their annual report a statement of the receipts and disbursements thereof. 
8. It shall be the duty of the Director of the said Experiment Station, in person or by deputy, to take samples for analysis from any lot or packages of any fertilizer or manure which way be in the possession of any dealer in the State, and he is hereby authorized to prescribe and enforce such rules and reguiations as he may deem necessary to carry fully into effect the true intent and meaning of this act; and any agriculturist, purchaser of any fertilizer or manure in this State may take a sample of the same under the rules and regulations of the Director of the said Experiment Station and forward the same to him analysis, and if the Director has reason to believe that the manutacturer of, or dealer in, such fertilizer or manure has made any 1alse or fradulent representations in regard to said fertilizer or manure, he shall cause the said sample to be analyzed free of charge, and certify the same to the person forwarding the san:ple. And it shall be the duty of every agent and dealer in fertiiyers or manures, within the meaning of this act, in this State, when ordering consignments thereof, to promptly notify the Director of the said Experiment Station of the amount ordered, and the place and approximate time of delivery of said fertil:zers and manures.

9. Said Director shall also publish by bulletin, the brant, rame and location of the manufacturer and chemical analysis of every fertilizer or manure analysed or caused to be analyzed py him. Said last publication to be made, if practicable, before the time at which said fertilizer is to be applied to the soil.

10. Any manufacturer or vendor of any fertilizer or manure who shall sell or offer or expose for sale any fertilizer or manure without haring previously complied with the provisions of this act as hereinbefore set forth, shall be deemed guilty of a misdemeanor, and upon conviction thereof shall be fined not less than fifty nor more than five hundred dollars.

1. Any company, firm or corporation who shall wilfuliy remove from or deface or change any label. or tag, or brand affixed to any package of fertilizer under the provisions of this act before such fertilizer has been used for manurial purposes, or who shall sell such fertilizer without such label or tag being affixed thereto at the time of the sale, shall be deemed guilty of a misdemeanor, and upon conviction thereof, shall be fined not les's than ten nor more than fifty dollars for each offense.

12. Any company, firm or person who shall remove from or cause to be removed from any package of commercial fertilizer or manure any statement. label or tag affired thereto under the pronisions of this act, and affix or cause the same to be affixed to any other package of commercial fertilizer or manure, shall 
be deened guilty of a misdemeanor, and upon conviction therecf sliall be fined not less than ten nor more than fifty dollars for 'ach offense.

13. Any company, firm or person riolating any of the provisions of this act. or who fails to comply with any of the? requirements of this act. shall be deemed guilty of a misdemeanur. and upon conviction thereof, shall, when no other penalty is nreesribed. be fined not less than ten nor more than one hunired dollars for each offense. But this act shall not be construed to apply to any one who manufactures fertilizers for his own use and not for sale.

14. The Director of said Experiment Station shall report to the prosecuting attorney of the county where the offense wis committed all violations of the provisions of this act, and failure to comply therewith, and a copy of any label. statement or tag required to he filed with said Director or prepared by him, and any analysis made or cansed to be made by him. when duly eertified hy said Director, shall be received in evidenee in any prosecution or suit for any violation of the provisions of this act.

I. Harry Shaw. Clerk of the House of Delegates of West Virginia. herehy certify that the paper hereto anuexed is a true copy of an Act of the Tegislature of West Virginia passed on the second day of Febmury, 1901, and approved by the Governor on the ninth day of February. 1901; and that the same takes efforet ninety days from the date of its passage.

Given under my hand this Nareh 20th, 1901.

HARRY SHIT, . . Clerk of the House of Delegates. 

